
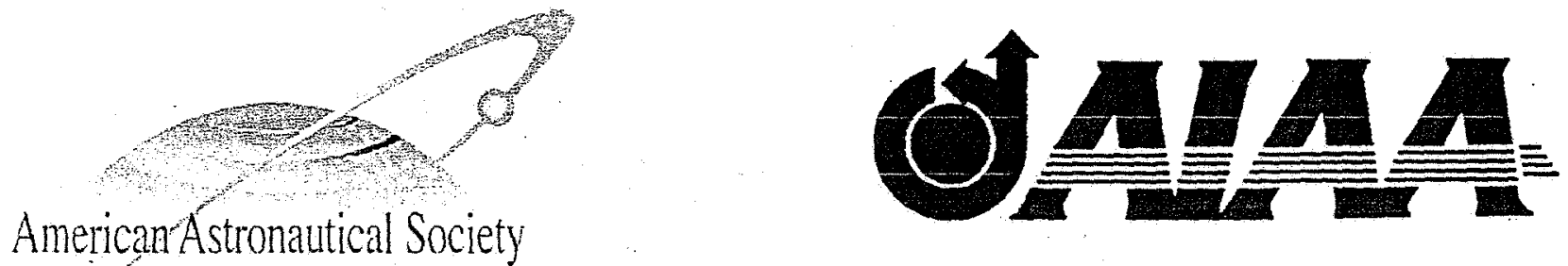

\title{
Multi-Body Orbit Architectures for Lunar South Pole Coverage
}

\author{
D. J. Grebow, M. T. Ozimek, K. C. Howell
}

School of Aeronautics and Astronautics, Purdue University

West Lafayette, Indiana 47907-1282

\author{
D. C. Folta
}

NASA Goddard Space Flight Center

Greenbelt, Maryland 20771

\section{$16^{\text {th }}$ AAS/AIAA Space Flight Mechanics Conference}

Tampa, Florida

January 22-26, 2006

AAS Publications Office, P.O. B@x 28130, San Diego, CA 92198 
Paper AAS 06-179

\title{
MULTI-BODY ORBIT ARCHITECTURES FOR LUNAR SOUTH POLE COVERAGE
}

\author{
D. J. Grebow, "M. T. Ozimek, K. C. Howell, ${ }^{\dagger}$ D. C. Folta ${ }^{\S}$
}

A potential ground station at the lunar south pole has prompted studies of orbit architectures that ensure adequate coverage. Constant communications can be achieved with two spacecraft in different combinations of Earth-Moon libration point orbits. Halo and vertical families, as well as other orbits near $L_{1}$ and $L_{2}$ are considered. The investigation includes detailed results using nine different orbits with periods ranging from 7 to 16 days. Natural solutions are generated in a full ephemeris model, including solar perturbations. A preliminary station-keeping analysis is also completed.

\section{INTRODUCTION}

One current focus of autonomous and manned exploration studies is the region near the south pole of the Moon due to the potential existence of frozen volatiles. ${ }^{1.2}$ Since the President's Vision for Space Exploration announcement in January 2004, NASA has indicated that water ice at the lunar poles may help facilitate exploration of the solar system. ${ }^{3}$ NASA's Exploration Communication and Navigation Systems (ECANS) Team, specifically the Lunar Communications and Navigation Systems (LCNS) group, is interested in spacecraft architectures for communications with ground stations on the lunar surface. Such a ground station on the Moon requires a system of satellites that is always in view of the Earth and that can provide constant communications between the lunar surface and the Earth. Various ideas are now being considered to support this concept. On a broad scale, for example, NASA's Living with a Star (LWS) program was created to learn more about the Sun-Earth system. ${ }^{4}$ The initiative inclided the possible investigation of north and south "pole-sitters" for constant Earth atmospheric monitoring and surveillance. Such ideas may be adaptable to the Moon for feasible south pole architectures. Studies of satellite constellations in Earth orbit are also being examined for application to the Moon.

The potential use of libration point orbits for lunar south pole coverage has not yet been fully investigated. However, an infinite variety of periodic solutions exist in the restricted three-body problem. Many of these families of orbits have been studied extensively by engineers, mathematicians, and astrophysicists (including Szebehely, ${ }^{5}$ Zagouras and Kazantzis, ${ }^{6}$ Breakwell and Brown, ${ }^{7}$ Robin and Markellos, ${ }^{8}$ Howell and Breakwell, ${ }^{9}$ and Dichman, Doedel, and Paffenroth ${ }^{10}$ ). Due to the nature of the solutions, some useful architectures for lunar south pole coverage may exist within the context of the threebody problem. Libration point orbits are investigated here to create architectures for coverage of the lunar south pole with only two spacecraft. The initial design phase originates in the Circular Restricted ThreeBody Problem (CR3BP). Vertical orbits, first introduced by Moulton ${ }^{11}$ in 1920, as well as other $L_{1}$ and $L_{2}$. families of orbit are investigated. The families are computed via a variable-time targeting scheme. ${ }^{7}$ Orbits are selected from these families based on (i) time to complete one full period; and, (ii) feasibility for lunar south pole coverage. Once the specific architecture is selected, the orbit is discretized into a series of patch

* Graduate Student, School of Aeronautics and Astronautics, Purdue University, Grissom Hall, 315 North Grant St., West Lafayette, Indiana 47907-1282; Student Member AIAA.

Hsu Lo Professor of Aeronautical and Astronautical Engineering, School of Aeronautics and Astronautics, Purdue University, Grissom Hall, 315 North Grant St., West Lafayette, Indiana 47907-1282; Fellow AAS; Associate Fellow AIAA.

\$ Senior Flight Dynamics Engineer, Bldg 11, Room S116, NASA Goddard Space Flight Center, Greenbelt, Maryland 20771: Senior Member AIAA. 
points. With modifications to a corrections scheme posed by Wilson and Howell, ${ }^{12}$ the patch points are then differentially corrected to meet both the desired time-of-flight and orbit periodicity requirements. The solutions are transitioned to the full ephemeris model, including solar perturbations, using the Purdue Software Package GENERATOR. ${ }^{13}$ Since the announcement of the near-term space exploration agenda, Ely and Lieb ${ }^{14,15}$ have investigated the placement of a system of satellites to support a south pole station and global orbital constellations using a two-body model with the gravitational perturbations of a third body as well as solar radiation pressure.

The long-term station-keeping costs are also an important factor in determining the feasibility of these systems. In 1971, Farquhar ${ }^{16}$ first examined the use of halo orbits to maintain a continuous communications link between the far side of the Moon and the Earth. His study includes a communications architecture and station-keeping costs. Later, extensive work on optimal station-keeping strategies using Floquet modes for halo orbits in the Earth-Moon system was completed by Simó et al. ${ }^{17,19}$ In this approach, the unstable subspace that is available from dynamical systems theory is used to develop a station-keeping strategy. Station-keeping analyses for Earth-Moon halo orbits were also completed by Howell and Pernicka, ${ }^{19}$ Howell and Keeter, ${ }^{20}$ and Gómez et al. ${ }^{21}$ Most recently, Scheeres et al. ${ }^{22}$ and Renault and Scheeres $^{23}$ have investigated the generalized optimal placement of statistical control maneuvers applied to orbits in the Earth-Moon restricted three-body problem. The orbits in these studies are typical of those that might be used for lunar coverage and also provide an additional benchmark for the station-keeping costs. Adapting models created by Folta and Vaughn, ${ }^{34}$ the patch points that define a libration point orbit from GENERATOR are targeted in Satellite Tool $\mathrm{Kit}^{\text {(i) }}$. Then, a facility is placed near the south pole of the Moon and access times between the facility and the dual spacecraft chain is analyzed. Thus, a preliminary station-keeping analysis in STK is accomplished.

\section{PERIODIC ORBITS AND THE CIRCULAR RESTRICTED THREE-BODY PROBLEM}

The Circular Restricted Three-Body Problem (CR3BP) describes the motions of three bodies subject to their mutual gravitational forces. The primary gravitational bodies move about their barycenter on circular paths. The third body, i.e., the spacecraft, is assumed to possess negligible mass in comparison to the primaries. The rotating $x$-axis is defined along the vector between the primaries; $z$-axis is parallel to the angular velocity vector associated with the Keplerian primary orbits. Then, the usual barycentric system of equations describing the motion of the third body are written

$$
\begin{aligned}
& \ddot{x}=2 \dot{y}+x-\frac{(1-\mu)(x+\mu)}{d^{3}}-\frac{\mu(x-(1-\mu))}{r^{3}}, \\
& \ddot{y}=-2 \dot{x}+y-\frac{(1-\mu) y}{d^{3}}-\frac{\mu y}{r^{3}} \\
& \ddot{z}=\frac{-(1-\mu) z}{d^{3}}-\frac{\mu z}{r^{3}}
\end{aligned}
$$

where $x, y$, and $z$ are the components of the third body's position relative to the rotating, barycentric frame. The mass parameter is $\mu$; then, $d$ and $r$ are the relative distances between the third body and the first and second primary, respectively. The equations are nondimensional, where the characteristic quantities are the total mass, the distance between the primaries, and the magnitude of the system angular velocity.

The state-transition matrix, $\Phi\left(t_{f}, t_{0}\right)$, associated with eq. (1), is also available. By analyzing endpoint variations, $\Phi\left(t_{f}, t_{B}\right)$ can be employed in an iterative process to yield the exact initial conditions for periodic motion in the CR3BP. From a first-order Taylor series expansion, the general form of a timevarying targeting scheme for convergence becomes 


$$
\left\{\begin{array}{l}
\delta \vec{R}_{f} \\
\delta \vec{V}_{f}
\end{array}\right\}=\left[\begin{array}{l:l}
\Phi\left(t_{f}, t_{0}\right) & \dot{\bar{R}} \\
& \dot{\bar{V}}
\end{array}\right]\left\{\begin{array}{c}
\delta \vec{R}_{0} \\
\delta \vec{V}_{0} \\
\delta\left(t_{f}-t_{0}\right)
\end{array}\right\},
$$

where $\vec{R}=\{x y z\}^{r}$ and $\vec{V}=\{\dot{x} \dot{y} \dot{z}\}^{T}$. The columns of $\Phi\left(t_{f}, t_{0}\right)$ are associated with the desired control parameters, i.e., $\delta \vec{R}_{0}, \delta \vec{V}_{0}, \delta\left(t_{f}-t_{0}\right)$, and the rows correspond to the end-point constraint parameters. ${ }^{7}$ The control parameters are iteratively updated by variations in the initial state until the end-point constraints are fully satisfied. Furthermore, once a single periodic solution is determined, neighboring solutions in the same family are computed using a method of continuation over an additional constraint parameter. The method is extrapolated to determine complete families of periodic orbits.

\section{Halo Orbits, Vertical Orbits, and Other $L_{1}$ and $L_{2}$ Families of Periodic Orbits}

Using the targeting scheme, families of $L_{1}$ and $L_{2}$ periodic halo and vertical orbits are quickly generated. In addition, a family of orbits that bifurcates from a near-rectilinear halo orbit is also obtained. Specific members of the orbit families are isolated based on both the communications instrument and subsurface constraints, bounding the useful range of orbits within a family to those with lunar altitudes between 50 and $100,000 \mathrm{~km}$.

A number of $L_{1}$ and $L_{2}$ southern halo orbits appear in Figure 1. These two families are particularly effective in this problem since the motion is almost always within line-of-sight to the Earth. Most recently, the halo orbit families have been thoroughly investigated by Farquhar, ${ }^{16}$ Breakwell and Brown, Howell and Breakwell, ${ }^{9}$ as well as Gómez et al. ${ }^{25}$ The halo orbits investigated here resemble both the traditional "halo" shape and the highly "elliptic", near-rectilinear motion with a passage very close to the Moon. For almost the entire period of the motion, a spacecraft in any near-rectilinear halo orbit possesses a line-ofsight to the lunar south pole.
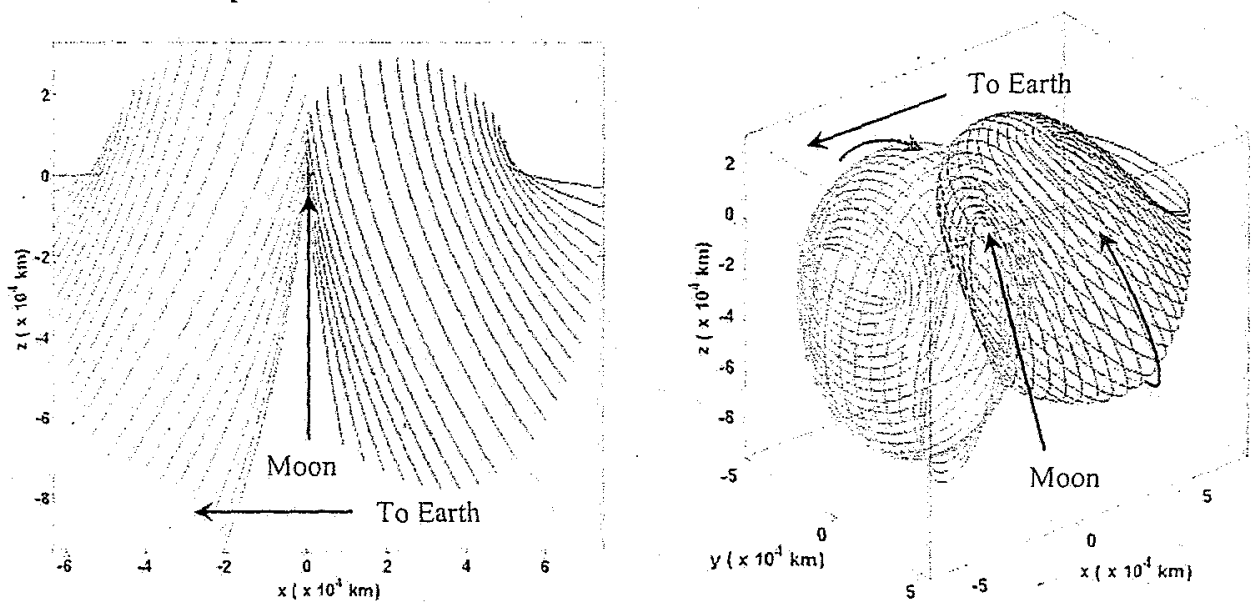

Figure 1 Southern Halo Orbit Families: Earth-Moon $L_{1}$ (Orange) and $L_{2}$ (Blue); Moon Centered, Rotating Reference Frame

Vertical orbits are doubly symmetric orbits near the libration points and, in the $y-z$ projection, resemble a "figure-8" in shape as seen in Figure 2. Since Moulton" highlighted their existence in 1920, the nonlinear vertical orbits have been extensively investigated by Zagouras and Kazantzis ${ }^{6}$ and most recently by Dichman et al. ${ }^{10}$ The complete families are attainable using a method of continuation with a targeting scheme based on eq. (2). Large amplitude $L_{1}$ vertical orbits terminate when they become exactly vertical, while large amplitude $L_{2}$ vertical orbits encompass both primaries. However, only vertical orbits with 
altitudes less than $100,000 \mathrm{~km}$ are feasible for coverage of the lunar poles. These orbits bend toward both the north and south poles of the Moon, a favorable characteristic for polar coverage.
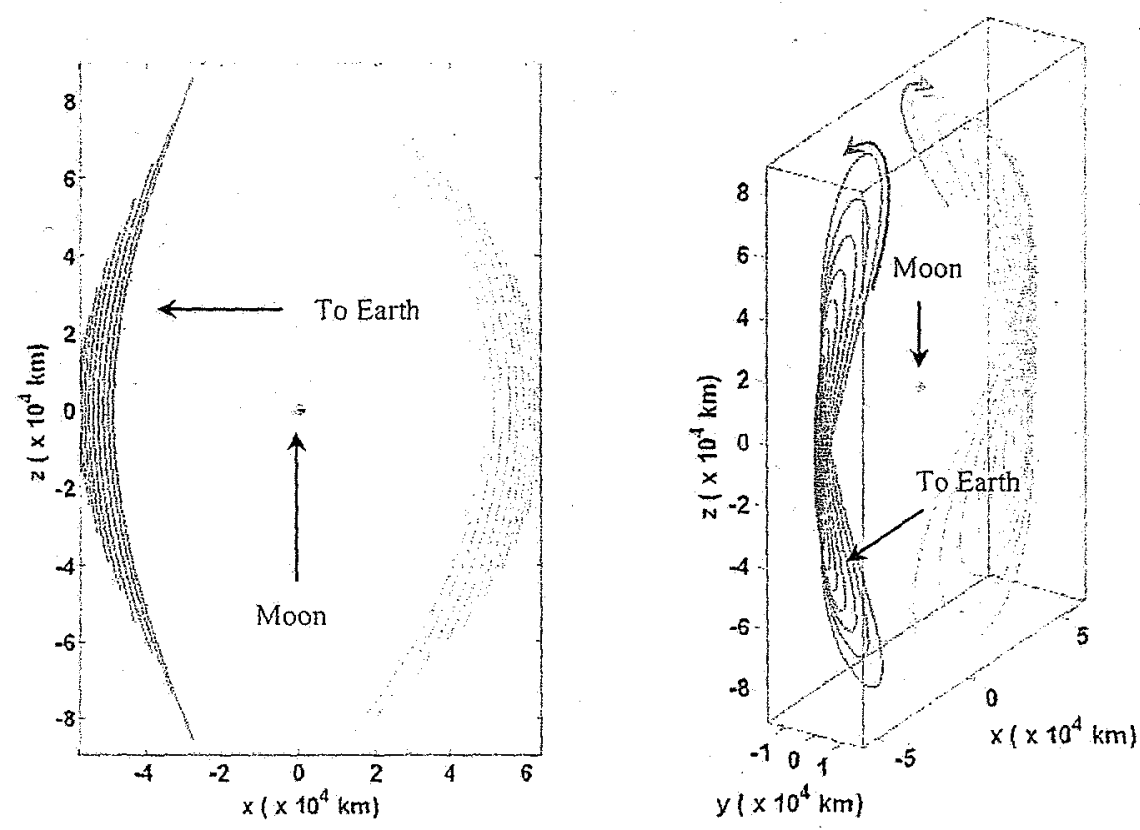

Figure 2 Vertical Orbit Family of Interest: Earth-Moon $L_{1}$ (Magenta) and $L_{2}$ (Cyan); Moon Centered, Rotating Reference Frame

An additional family also incindes orbits that remain in view of the lunar south pole for significant intervals of time. Some of these orbits possess characteristics similar to the near-rectilinear halo orbits. The orbits bifurcate from a 6 -day near-tectilinear $L_{2}$ halo orbit and might be described as a "butterfly" shape. (See Figure 3.) Comparable motions around the smaller primary have been documented by Robin and Markellos. ${ }^{8}$ Similar to vertical orbits, the motion in a butterfly orbit resembles a "figure- 8 ", however, these orbits wrap around both the near and far side of the Moon, such that a direct line-of-sight to the lunar south pole exists for nearly the entire orbital period.
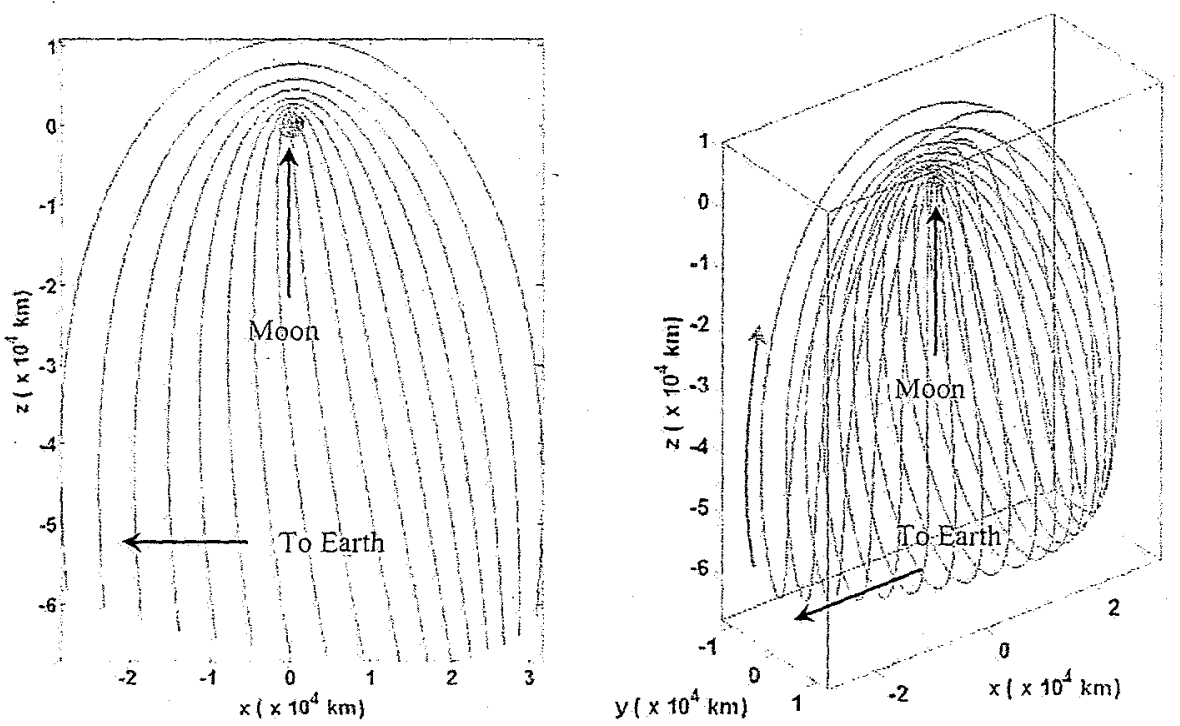

Figure 3 Southern $L_{2}$ Butterfly Orbit Family; Moon Centered, Rotating Reference Frame 


\section{Orbit Selection Criteria}

The time to complete one full period is a useful selection parameter in the initial design phase. Let the maximum excursion distance, as shown with the example halo orbit in Figure 4 (bottom right), be defined as the maximum $x$-distance for each orbit in the Moon centered, rotating frame. Orbital periods are plotted against maximum excursion distance during initial design selection, as indicated in Figure 4 (left). Suitable regions for the production of feasible architectures occur when the orbital periods are commensurate. One such region consists of orbits in $L_{1}$ and $L_{2}$ halo families sharing periods between 7.9 and 12.2 days. An example that exhibits feasible south pole coverage consists of a 12-day $L_{1}$ and 12-day $L_{2}$ halo orbit combination, illustrated by the black dashed line in Figure 4. Another region with commensurate combinations consists of orbits with a ratio of periods equal to $2: 1$, that is, one period is exactly twice that of the other. Note that $L_{2}$ halo orbits with periods between 6.0 to 7.2 days exhibit this behavior with the entire $L_{2}$ butterfly orbit family. This is not actually surprising when the shapes of the orbits are viewed in Figures 1 and 3. An example from this region consists of a 14-day $L_{2}$ butterfly orbit and a 7-day $L_{2}$ halo orbit combination, as noted by the two red dashed lines in Figure 4 . The information in Figure 4 serves as a basis for the determination of many other commensurate orbit combinations that lead to complete south pole coverage.
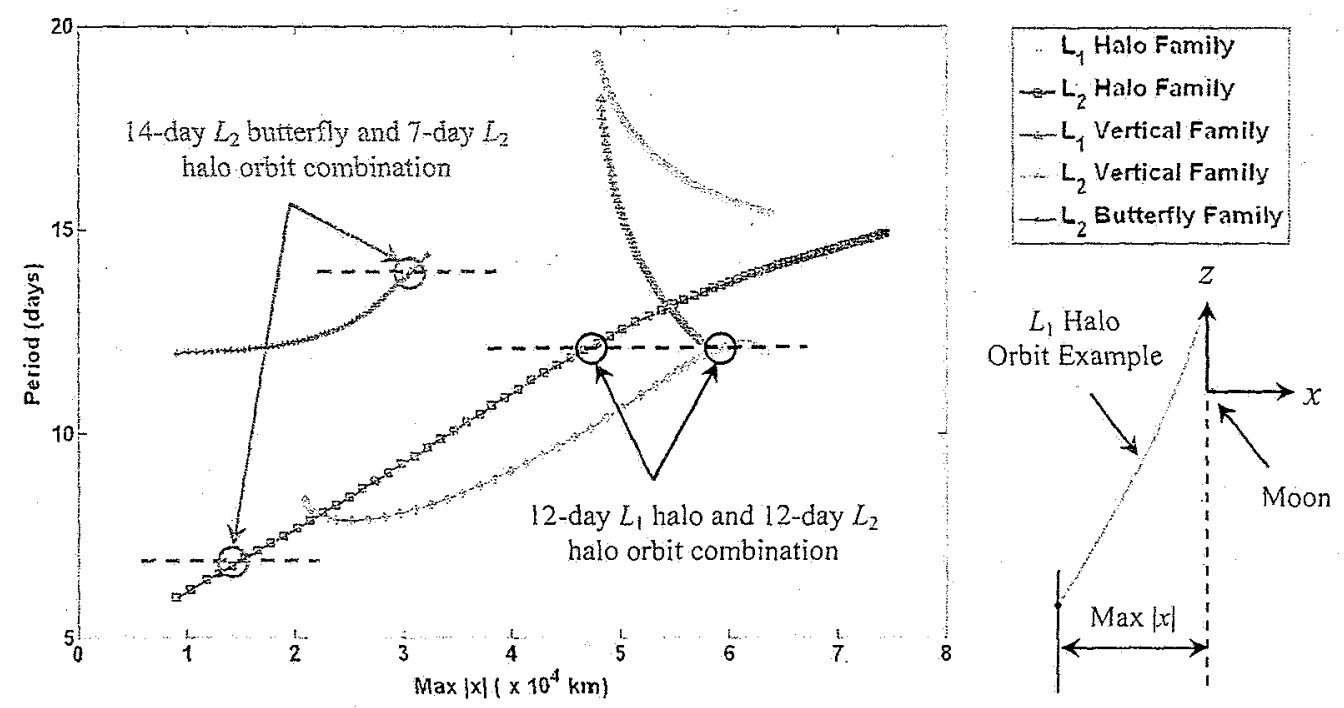

Figure 4 Period versus Maximum $x$-Distance from the Moon (Left); Definition of Maximum $x$-Distance (Bottom Right)

Also useful for design purposes is the stability index, $v$. The stability index, corresponding to one orbit period $T$, is defined as

$$
v=\frac{1}{2}\left(\lambda_{\max }+\frac{1}{\lambda_{\max }}\right)
$$

where $\lambda_{\max }$ is the maximum eigenvalue from the monodromy matrix, $\Phi(T+t, t)$, computed at the end of one revolution. A stability index of one indicates a stable orbit, whereas stability indices greater than one reflect instability. Of course, a large stability index indicates a divergent mode that departs from the vicinity of the orbit very quickly. Generally, the stability index is directly correlated to the station-keeping costs and is inversely related to transfer costs. The stability indices for orbits from the various families appear in Figure 5 as functions of maximum excursion distance from the Moon. In general, the stability index increases with distance from the Moon. 

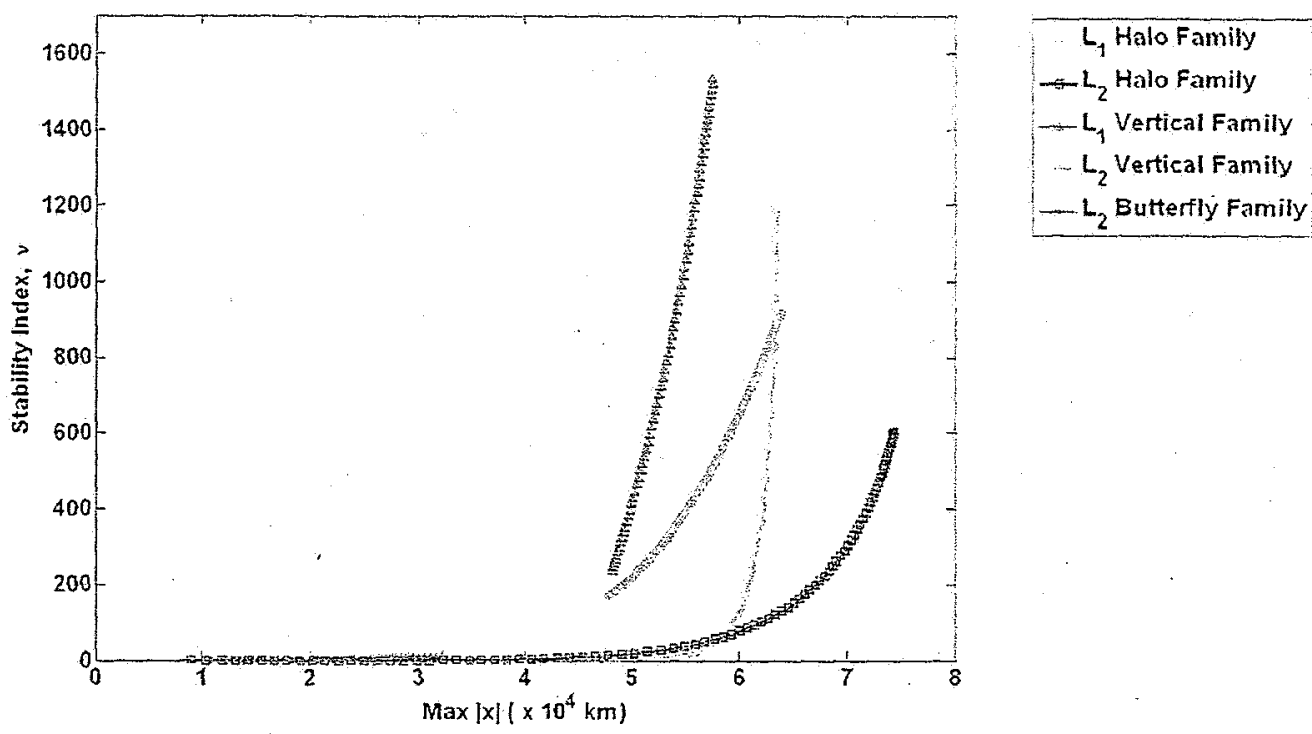

Figure 5 Stability Index versus Maximum Distance from the Moon

Using periodicity and stability criteria, orbits from Figures $1-3$ are selected for use in a coverage scenario. Either a single orbit is selected and two spacecraft are placed in the same orbit (but out-of-phase), or a unique orbit is selected for each spacecraft such that their periods are commensurate. When an orbit with a precisely defined period is desired, a modified two-level differential corrections scheme accepts the patch points of a previously generated, neighboring orbit as an initial guess and iterates to generate an orbit with the specified period. When this process is complete for all desired orbits, patch points for multiple revolutions are added to develop a baseline 180-day mission. Once the final trajectories are obtained, the initial time corresponding to one of the two spacecraft is phase shifted by a half-period, thus allowing the greatest chance for complete coverage of the lunar south pole.

\section{The Modified Two-Level Differential Corrector}

In 1988, Howell and Pernicka ${ }^{26}$ used a two-level differential corrections scheme to determine continuous, quasi-periodic motion near the libration points in the CR3BP. The two-level process has more recently been modified to include multiple flight regimes by Wilson and Howell ${ }^{12}$ and Hughes, Cooley, and Guzman. ${ }^{27}$ The first level of the corrections scheme employs eq. (2) to target positions defined in terms of discretized state vectors, or patch points, along the desired orbit. The second phase, or level, computes small variations in the patch point positions and times to minimize the velocity discontinuities at each of the first level patch points. Iterations continue until the intermediate velocity variations $\delta \bar{V}$ are reduced to zero and a natural solution is identified. The current work utilizes the targeter created by Howell and Pernicka, adapted to incorporate constraints as posed by Wilson and Howell.

Using Wilson's notation, there exists a state-relationship matrix, M, such that the smallest Euclidean norm yields the new variations in position $\left(\delta \vec{R}_{i}\right)$ and time $\left(\delta t_{i}\right)$ corresponding to the $t^{\text {th }}$ patch point, i.e., 


$$
\left\{\begin{array}{c}
\delta \vec{R}_{1} \\
\delta t_{1} \\
\vdots \\
\delta \vec{R}_{n} \\
\delta t_{n}
\end{array}\right\}=[\mathbf{M}]\left([\mathbf{M}][\mathbf{M}]^{T}\right)^{-1}\left\{\begin{array}{c}
\delta \vec{V}_{2} \\
\vdots \\
\delta \vec{V}_{n-1}
\end{array}\right\}
$$

where $\delta \vec{V}_{i}$ represents the internal discontinuity in the velocity vector (to be minimized) from the first level of the corrections procedure for $n$ patch points. The components of the state-relationship matrix $M$ are derived from the linear variational equations relating consecutive patch point states.

The method can also be adapted to compute $\delta \vec{R}_{i}$ and $\delta t_{i}$ subject to additional constraints. The periodicity constraint is detailed in Marchand et al. ${ }^{28}$ and one example of the implementation appears in Marchand and Howel1. ${ }^{29}$ For this analysis, the orbit must be periodic, and a specified time-of-flight, TOF, condition must be satisfied as well. Therefore, eq. (4) becomes

$$
\left\{\begin{array}{c}
\delta \vec{R}_{1} \\
\delta t_{1} \\
\vdots \\
\delta \vec{R}_{i} \\
\delta t_{n}
\end{array}\right\}=\left[\mathbf{M}^{*}\right]\left(\left[\mathbf{M}^{*}\right]\left[\mathbf{M}^{*}\right]^{r}\right)^{-1}\left\{\begin{array}{c}
\delta \vec{V}_{2} \\
\vdots \\
\delta \bar{V}_{n-1} \\
\vec{V}_{n}-\vec{V}_{1} \\
\vec{R}_{n}-\vec{R}_{1} \\
T O F-\left(t_{n}-t_{1}\right)
\end{array}\right\}
$$

where $\mathrm{M}^{*}$ also includes the partial derivatives of $\vec{V}_{n}-\vec{V}_{1}, \vec{R}_{n}-\vec{R}_{1}$, and TOF $-\left(t_{n}-t_{1}\right)$ with respect to position and time such that

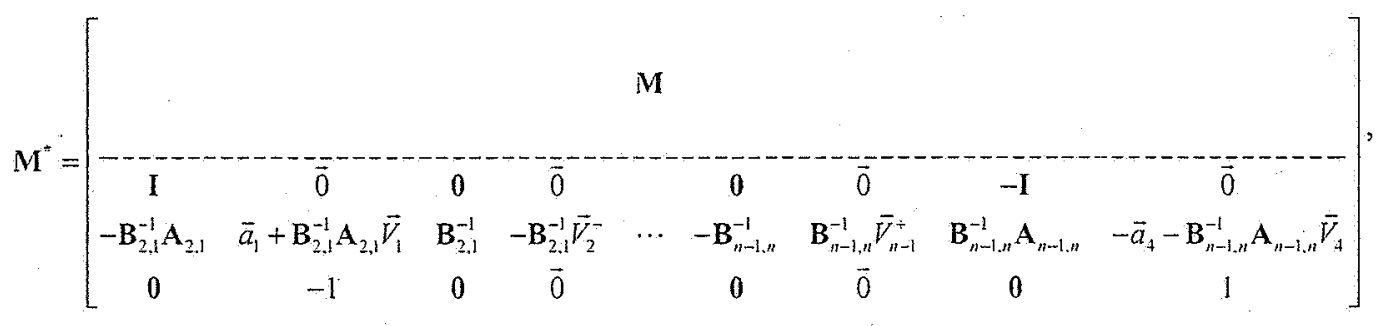

$$
\text { for } \Phi\left(t_{2}, t_{1}\right)=\left[\begin{array}{c:c}
\mathbf{A}_{2,1} & \mathbf{B}_{2,1} \\
\hdashline \mathbf{C}_{2,1} & \mathbf{D}_{2,1}
\end{array}\right] \text { and } \Phi\left(t_{n-1}, t_{n}\right)=\left[\begin{array}{c:c}
\mathbf{A}_{n-1 . n} & \mathbf{B}_{n-1 . n} \\
\hdashline \mathbf{C}_{n-1, n} & \mathbf{D}_{n-1, n}
\end{array}\right] \text {. }
$$

Note that $\bar{a}_{i}$ indicates an acceleration vector. Thus, $\delta \vec{V}_{i}, \vec{V}_{n}-\vec{V}_{1}, \vec{R}_{n}-\vec{R}_{1}$, and TOF $-\left(t_{n}-t_{1}\right)$ are all minimized iteratively until the periodic orbit that satisfies the required time-of-flight condition is obtained.

\section{Invariant Manifold Theory and the Unstable Subspace}

The unstable subspace is available from analysis of the phase space and is useful in a preliminary station-keeping analysis. For $n$ patch points along a periodic orbit, where the first and last patch points coincide, the monodromy matrix for the $i^{\text {th }}$ point along the orbit is computed as

$$
\Phi\left(T+t_{i}, t_{i}\right)=\left[\Phi\left(t_{i \neq n}, t_{i+n-1}\right)\right]\left[\Phi\left(t_{i+n-1}, t_{i+n-2}\right)\right] \cdots\left[\Phi\left(t_{i+2}, t_{i+1}\right)\right]\left[\Phi\left(t_{i+1}, t_{i}\right)\right],
$$


where $T=t_{i+n}-t_{i}$ is the time to complete one full period. Let the six-dimensional vector $\hat{Y}_{i}^{1{ }^{\prime}}{ }^{\prime}$ represent the eigenvector associated with the unstable mode from $\Phi\left(T+t_{i}, t_{i}\right)$ such that it can be written in terms of three-dimensional components $\vec{R}_{i}^{W_{*}^{\prime}}$ and $\vec{V}_{i}^{W{ }^{\prime \prime}}$ as

$$
\hat{Y}_{i}^{I^{\prime \prime}}=\left\{\begin{array}{l}
\vec{R}_{i}^{W_{a}} \\
\vec{V}_{i}^{W_{1 *}}
\end{array}\right\} .
$$

Then the unstable direction is represented by

$$
\dot{X}_{i}^{W_{t I}}=\frac{\hat{Y}_{i}^{W_{u}}}{R_{i}^{W W_{u}}}
$$

A small perturbation in the unstable direction, $\bar{X}_{i}^{\text {Hi }}$, places the spacecraft on an unstable manifold departing the vicinity of the reference solution. The perturbing terms are represented by

$$
\left\{\begin{array}{l}
\delta \bar{R}_{i} \\
\delta \bar{V}_{i}
\end{array}\right\}=d \cdot \vec{X}_{i}^{W^{\prime}},
$$

where $d$ is an initial displacement in the unstable direction. The unstable manifold is obtained by perturbing each patch point state by $\pm\left\{\delta \vec{R}_{i}^{T} \quad \delta \vec{V}_{i}^{T}\right\}^{r}$ and integrating forward and backward in time. This information is useful for station-keeping analysis in general. In this study, it was incorporated into a Satellite Tool Kit (STK) analysis.

\section{ADVANCED MODELING IN GENERATOR AND SATELLITE TOOL KIT $\$$}

All orbits are initially designed under the assumption that a spacecraft is subject only to the gravitational forces of the Earth and Moon in the CR3BP. High fidelity software that more accurately represents the force models and perturbing motions is desired to ensure complete coverage of the lunar south pole and provide preliminary station-keeping costs. For this reason, the Purdue software GENERATOR and AGI's Satellite Tool $\mathrm{Kit}^{\mathbb{R}}$ (STK) are used to precisely model and analyze all coverage schemes of interest.

\section{Full Ephemeris Modeling with Purdue Software Package GENERATOR ${ }^{13}$}

The Purdue software package GENERATOR is a mission design tool that is based on multi-body equations of motion including solar perturbations. Preliminary baseline trajectories can quickly be determined within the context of the two-, three-, or four-body problem. Any number of bodies and ephemeris information can be incorporated as desired (other forces, control schemes, and design components are available but not employed here). For this application, the 180-day baseline orbits acquired by using the modified two-level differential corrector in the CR3BP are transferred to the GENERATOR full ephemeris model. The patch point velocity discontinuities are minimized within GENERATOR and a modified orbit emerges. The resulting coverage schemes are initially analyzed by examination of the $z$-displacement of each spacecraft at the same instant of time. The z-displacement reflects the out-of-plane component of the position vector. The potential to maintain line-of-sight to the lunar south pole exists only if at least one of the spacecraft is below the Earth-Moon fundamental plane $z=0$ at all times. Consider two spacecraft in a single $L_{1}$ halo orbit. A typical two-spacecraft coverage scheme is achieved by displacing the motion of each spacecraft by a half period. Thus, the two spacecraft are then phase shifted in the $L_{1}$ halo orbit and the $z$-displacement of each spacecraft as a function of time appears in Figure 6 . The dashed line highlights the $z$-value at which the two spacecraft possess a common 


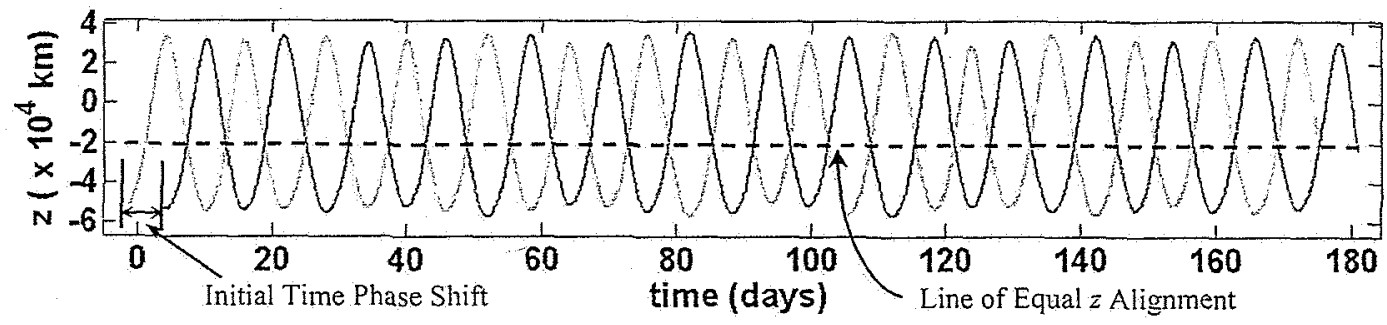

Figure 6 Sample Coverage Scenario: $Z$-Displacement from Rotating Reference Frame for Two Spacecraft in 12-Day $L_{1}$ Halo Orbits

$z$-component but are moving in opposite directions along the orbit. The dashed line in Figure 6 demonstrates that the $z$-crossing occurs significantly below the fundamental plane, ensuring that at least one spacecraft is always within direct line-of-sight to the south pole. The first goal is to maximize the distance between the dashed line and the fundamental plane $(z=0)$. This initial step does not fully account for the actual position of the lunar south pole due the tilt and nutation of the rotation axes, but it provides an estimate for the line-of-sight coverage behavior over time. Additionally, analyzing the $z$-displacement in this way also offers a visual confirmation that the proper periodicity constraint is implemented correctly.

\section{Station-Keeping and Coverage Analyses in Satellite Tool Kit ${ }^{\circledast}$}

Station-Keeping. Once a baseline coverage scenario is constructed, potential station-keeping costs are investigated. Adapting a model created by Folta and Vaughn, ${ }^{24}$ patch points along the orbits from GENERATOR are targeted in Astrogator using Astrogator Connect. The propagator in the targeting sequence is an eighth order, full Runge-Kutta-Verner integrator with ninth order error control including solar perturbations. Targeting GENERATOR patch points in this slightly different model implies that small corrections are required at each point. In general, however, transitioning from GENERATOR to Astrogator results in less than $1 \mathrm{~m} / \mathrm{s}$ per year for these corrections. (Although these corrections could be removed with slight adjustment to the orbits, it was not deemed necessary for this preliminary analysis.)

A preliminary station-keeping analysis is constructed by modifying the targeting sequence in Astrogator Connect. The station-keeping algorithm for four patch points appears in Figure 7. Note that the green path represents the reference orbit as computed directly from GENERATOR. Along the green path, the patch points from GENERATOR are indicated as dots and are defined as the target points in

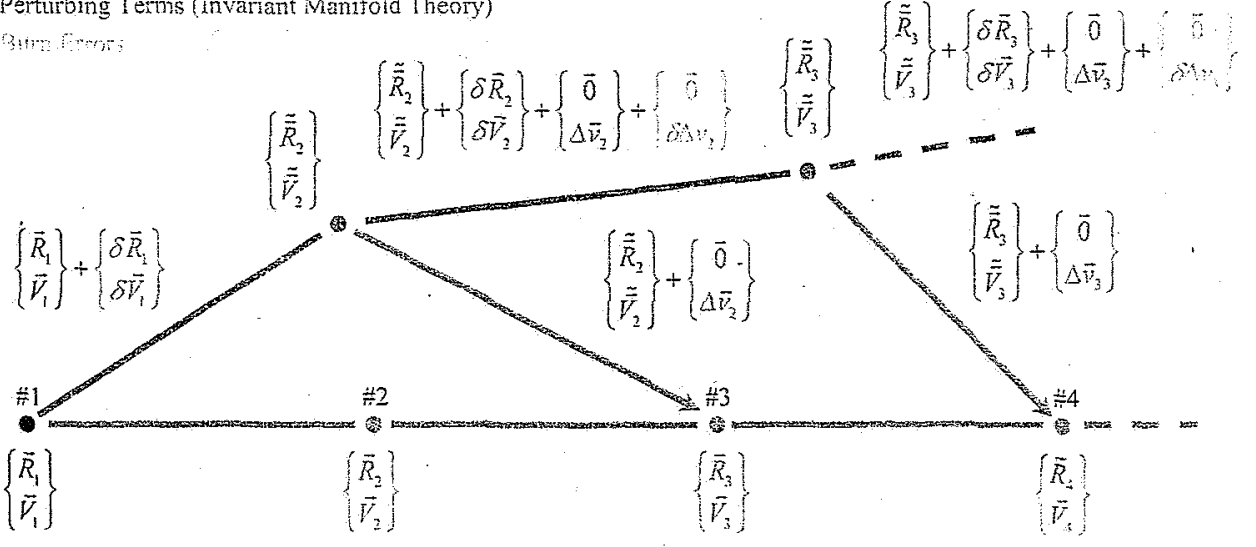

Figure 7 Schematic for Station-Keeping Algorithm 
Astrogator, i.e., $\left\{\begin{array}{cc}\vec{R}_{i}^{T} & \vec{V}_{i}^{T}\end{array}\right\}^{T}$. The vehicle is not actually associated with the reference state, of course. Perturbing the spacecraft into the unstable subspace and determining the maneuvers necessary to offset the error and maintain the orbit ultimately yields a good approximation of the maximum station-keeping cost. $^{17-23}$ So, the perturbations along the unstable direction are computed from eq. (11) and appear in magenta as $\left\{\begin{array}{ll}\delta \vec{R}_{i}^{T} & \delta \vec{V}_{1}^{T}\end{array}\right\}^{\tilde{T}}$. Recall that position and velocity perturbations are represented by the displacement $d$ along the unstable direction in eq. (11). These perturbations might represent navigation errors, for example, and the impact of such errors is most significant if they are in the unstable direction. The magnitude of $d$ is sized to be consistent with the magnitude of some average navigation $3 \sigma$ position errors; thus, the value might typically cover a range from 2 to $3 \mathrm{~km}$ in position. To account for slight differences between the unstable directions in the CR3BP and the full model, a baseline $3 \sigma$ position error of $5 \mathrm{~km}$ is used in this analysis. These perturbations $\left\{\delta \vec{R}_{1}^{T} \quad \delta \vec{V}_{1}^{T}\right\}^{T}$ are added to the initial state and propagation in Astrogator over the time interval necessary to reach point $\# 2$ moves the vehicle along a new actual path (in blue). Of course, the state associated with the spacecraft at the end of this propagation is actually $\left\{\begin{array}{ll}\tilde{\vec{R}}_{2}^{T} & \tilde{\bar{V}}_{2}^{T}\end{array}\right\}^{T}$. A correction maneuver $\left(\Delta \bar{v}_{2}\right)$ is computed via a targeting sequence in Astrogator to actually reach the next target point ${ }_{\# 3}$. (This $\Delta \vec{v}_{z}$ is representative of the cost to offset the error introduced via $\left.\left\{\begin{array}{ll}\delta \vec{R}_{1}^{T} & \delta \vec{r}_{1}^{T}\end{array}\right\}^{T}\right)$. The $\Delta \bar{v}_{2}$ from the targeting sequence is adjusted by $\pm 2 \%$ to include hot $(+)$ or cold $(-)$ burn errors $(\mathrm{A})$. However, to incorporate possible errors, perturbations along the unstable direction are

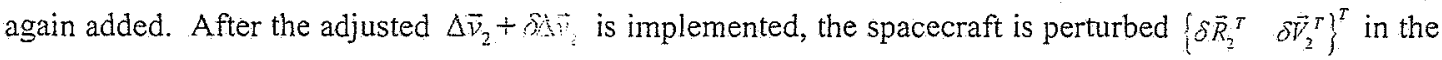
unstable direction and again propagated forward. The process then continues for a specified time interval (that is, a pre-determined number of target points). The total station-keeping cost is the sum of the $|\Delta \bar{v}|$ 's.

Coverage. A full lunar south pole coverage analysis is also available using STK. A facility is positioned at the south pole of the Moon and a chain is created between the two spacecraft. Assuming an omnidirectional communications link, the spacecraft is only accessible to the facility if it is within a direct lineof-sight. Intervals when the facility is unable to access either spacecraft are recorded. Furthermore, information from the facility regarding each spacecraft during the access times is available. For example, a plot of the elevation angle of each spacecraft from the facility as a function of time appears in Figure 8. The plot demonstrates that the desired coverage is achieved. From Figure 8, it is apparent that at least one spacecraft is always $15^{\circ}$ above the horizon as viewed from the facility.

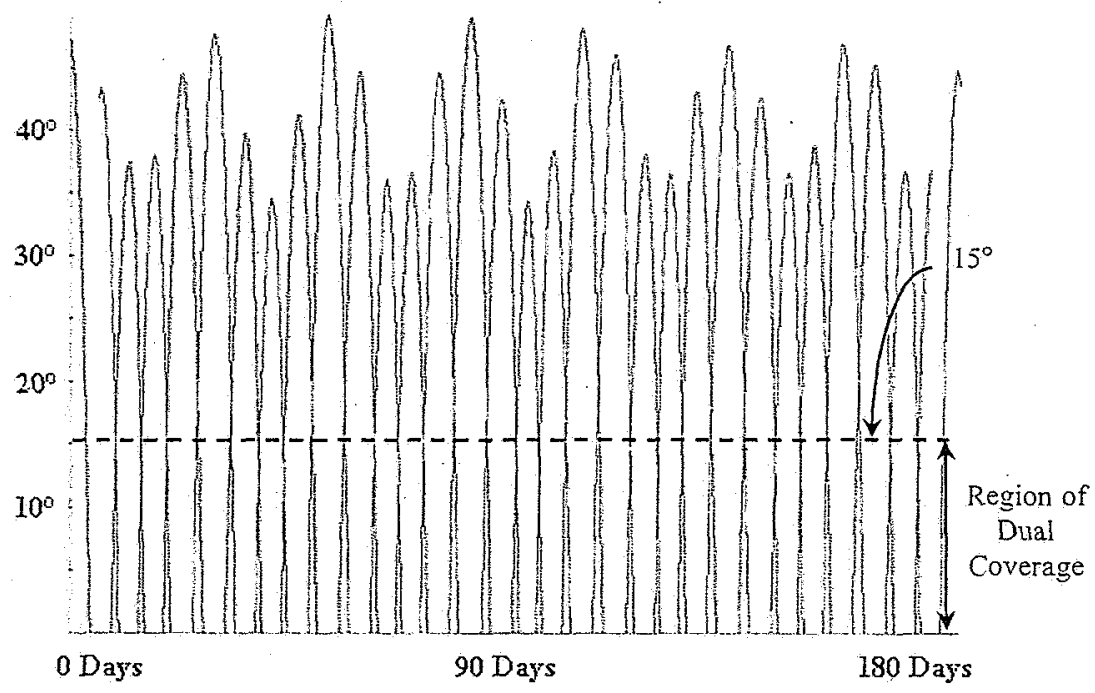

Figure 8 Elevation of Each Spacecraft Above the Horizon as Viewed from Lunar South Pole Facility for 12-Day $L_{1}$ Halo Orbit Scenario 


\section{ARCHITECTURES FOR LUNAR SOUTH POLE COVERAGE}

For this investigation, nine different orbits from the families represented in Figures 1-3 are selected based on their periods. (See Figure 4.) Coverage can be adequately ensured with two spacecraft in just one of these nine orbits by phasing the vehicles appropriately. However, as already noted, selecting orbits with commensurate periods allows for architectures with combinations of two different orbits for complete lunar south pole coverage. For example, rather than placing two spacecraft in the same 7-day $L_{2}$ near-rectilinear halo orbit, use one spacecraft in a 7-day $L_{2}$ near-rectilinear halo and the other vehicle in a 14-day $L_{2}$ butterfly orbit. The combination may provide more complete coverage. Possible orbits for use in combination to ensure lunar south pole coverage are represented in Table 1.

Table 1

POTENTIAL ORBITS FOR COVERAGE OF THE LUNAR SOUTH POLE

\begin{tabular}{cccc}
\hline Orbit Type & Libration Point & Period (days) & Stability Index \\
\hline Near-Rectilinear Halo & $L_{2}$ & 7.0 & 1.00 \\
Near-Rectilinear Halo & $L_{1}$ & 8.0 & 1.25 \\
Near-Rectilinear Halo & $L_{2}$ & 8.0 & 1.00 \\
Halo & $L_{1}$ & 12.0 & 60 \\
Halo & $L_{2}$ & 14.0 & 115 \\
Vertical & $L_{1}$ & 14.0 & 690 \\
Butterfly & $L_{2}$ & 14.0 & 245 \\
Vertical & $L_{1}$ & 16.0 & 370 \\
Vertical & $L_{2}$ & 16.0 & 515 \\
\hline
\end{tabular}

Sample Case: Two 12-Day $L_{1}$ Halo Orbits

One possible solution to the coverage problem employs two spacecraft in the same 12-day $L_{1}$ halo orbit. The lunar periapsis altitude for this orbit is approximately $36,500 \mathrm{~km}$. One spacecraft is phase shifted by a half-period, thus allowing the greatest chance for lunar south pole coverage, and the relative spacecraft positions appear in Figure 9. Fifteen revolutions are added for a baseline 180-day mission.

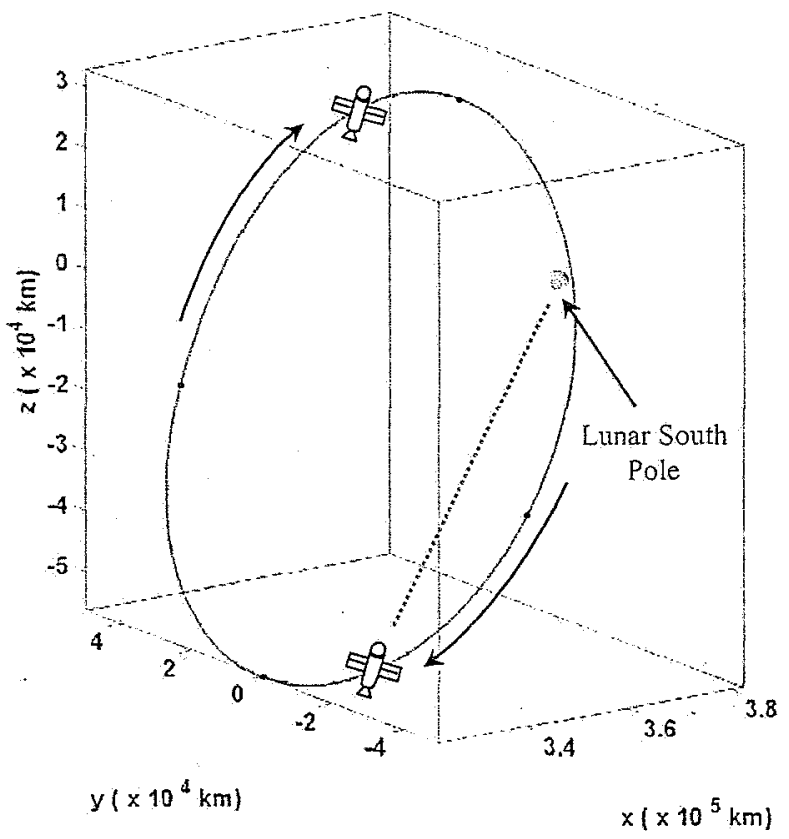

Figure 9 12-Day $L_{1}$ Orbit (Blue) and Patch Points (Black) from CR3BP; Earth-Moon Rotating, Barycentric Reference Frame

The orbits are transitioned to the full model in the Purdue software GENERATOR. Due to the prescribed phasing, at least one spacecraft is always $19,500 \mathrm{~km}$ below the Earth-Moon fundamental plane, 
ensuring coverage of the lunar south pole. (Recall Figure 6.) The orbits for the two spacecraft that result from GENERATOR appear in Figure 10 in both a Moon centered rotating system of coordinates and the Inertial Mean J2000 frame. Note that the trajectory of one spacecraft is plotted in blue; the motion of the other spacecraft appears in red. The quasi-periodic motion of the spacecraft is most apparent in the EarthMoon rotating frame where both orbit trajectories follow nearly the same path. Note that the patch point positions (black) have been adjusted for continuous motion in the full ephemeris model. In the Inertial Mean J2000 frame, the motion of the spacecraft appears to "umbrella" around the Moon.

Targeting these GENERATOR patch points in Astrogator (without any additional errors) using Astrogator Connect requires less than $0.41 \mathrm{~m} / \mathrm{s}$ over fifteen revolutions in total corrections for each spacecraft. The station-keeping analysis is performed by perturbing the spacecraft in STK using a $3 \sigma$ value of $5 \mathrm{~km}$ along the CR3BP unstable direction that is determined from the phase space analysis. The corresponding mean $3 \sigma$ velocity perturbation is $3.82 \mathrm{~cm} / \mathrm{s}$. To remain close to the nominal path, a maneuver is implemented every second patch point. Thus, for the baseline 180-day mission, each spacecraft implements a maneuver every six days of average size $1.11 \mathrm{~m} / \mathrm{s}$. The total maximum $|\Delta \vec{v}|$ for each spacecraft is $31.17 \mathrm{~m} / \mathrm{s}$ including the $2 \%$ burn errors.
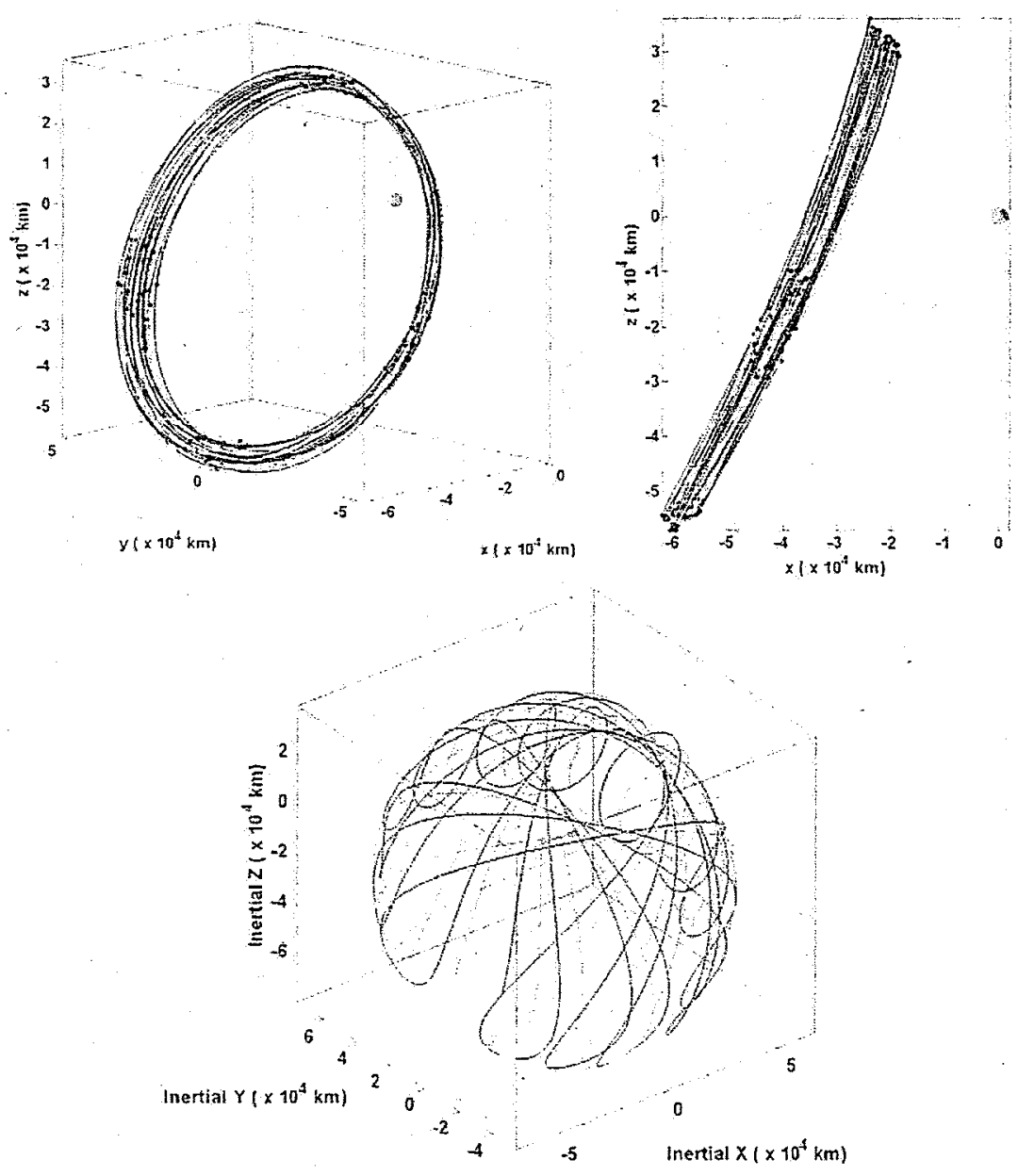

Figure 10 Two Phased Spacecraft in 12-Day $L_{1}$ Halo Orbits from Generator: Moon Centered, Rotating Reference Frame (Top Left), $X-Z$ Projection (Top Right); Inertial Mean $\mathbf{J} 2000$ Reference Frame (Bottom) 
A. facility is created at the lunar south pole and the access times between the facility and spacecraft chain are acquired in STK. (See Figure 11.) Note that an access beam (yellow) only appears when a spacecraft is within direct line-of-sight of the facility. As demonstrated in Figure 8, when the elevation angle of one spacecraft relative to the facility decreases to around $20^{\circ}$, the elevation angle of the line-ofsight vector to the other spacecraft appears above the horizon for complete access. In this way, one spacecraft trades access times with the facility while the other spacecraft passes over the northern hemisphere of the Moon. Therefore, even with the inclusion of lunar nutation and inclination with respect to the Earth-Moon fundamental plane, at least one spacecraft is always directly within line-of-sight of the south pole.

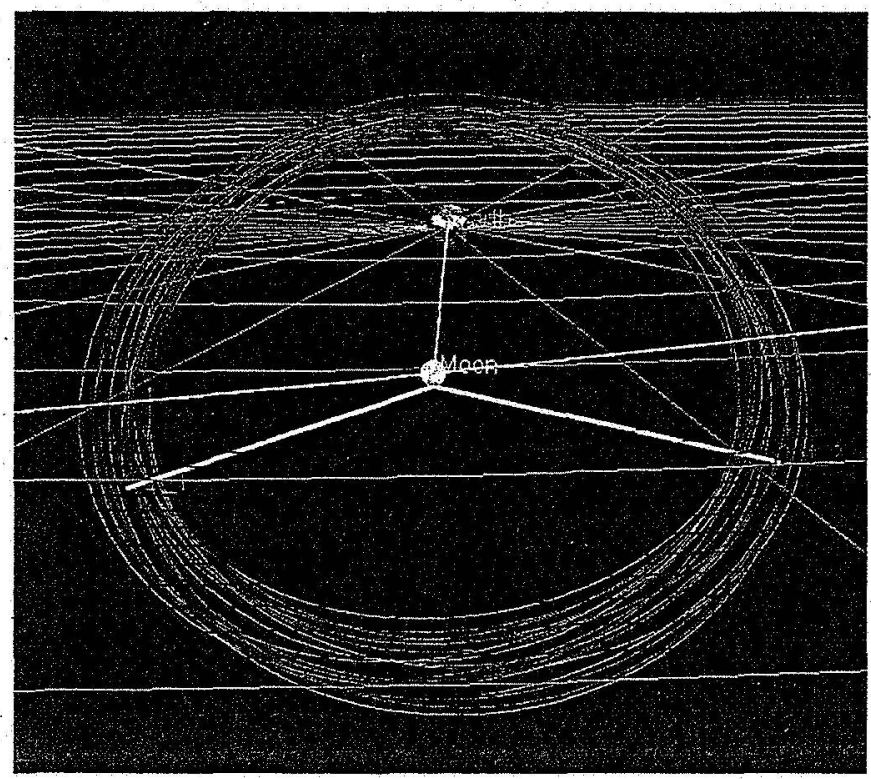

Figure 11 STK 12-Day $L_{1}$ Halo Orbits (Blue and Red) with Access Bearns (Yellow); Earth Centered, Rotating Reference Frame

\section{Other Combinations for Lunar South Pole Coverage}

Besides two spacecraft in the same 12-day $L_{1}$ halo orbit, other combinations of various orbits from Table 1 also yield viable coverage options. Observe that Table 1 also includes stability indices that are computed for each orbit. In general, smaller periapsis altitudes directly correlate to smaller stability indices. Also, smaller distances between the dashed line for equal $z$ alignment and the fundamental plane, as computed in GENERATOR, translate to smaller ranges over which the elevation angles of the two spacecraft allow simultaneous coverage scenarios in STK. A smaller range over the elevation angles reflects smaller time intervals over which the lunar south pole facility has access with both spacecraft.

The $L_{2} 7$-day halo orbit combination, with the orbits plotted in Figure 12a, utilizes only one halo orbit for both spacecraft. This near-rectilinear orbit passes approximately $2,750 \mathrm{~km}$ from the lunar surface at periapsis, $72,100 \mathrm{~km}$ at apoapsis altitude, and possesses a stability index of 1.00 . Analyzing the GENERATOR $z$-displacement components, the scenario results in a distance between the dashed line for equal $z$ alignment and the fundamental plane of $55,000 \mathrm{~km}$. (See Figure 12b.) Complete coverage of the facility occurs at all times, as observed in Figure $12 \mathrm{c}$, and both spacecraft simultaneously achieve access with the facility for elevation angles from $0^{\circ}$ to $65^{\circ}$. Noted in Figure 12a is a time when both spacecraft are accessed by the facility, i.e., both yellow access beams are active. Since the $L_{2} 7$-day halo orbit is nearrectilinear, the motion is similar to a highly elliptical two-body orbit. Therefore, during the majority of the orbital period both spacecraft are within direct line-of-sight of the lunar facility, leading to large periods of redundant coverage. 
The 8-day $L_{1}$ and $L_{2}$ halo orbit scenario depicts an orbit combination that utilizes halo orbits about two distinct libration points (Figures 13). Like the 7-day scenario, the 8-day $L_{1}$ and $L_{2}$ halo orbits offer low minimum altitudes at approximately $1,400 \mathrm{~km}$ and $6,000 \mathrm{~km}$, with apoapsis altitudes of $90,200 \mathrm{~km}$ and $76,900 \mathrm{~km}$, and stability indices of 1.25 and 1.00 , respectively. The GENERATOR analysis of Figure $13 \mathrm{~b}$ demonstrates that the minimum distance between the dashed line for equal $z$ alignment below the fundamental plane is $60,000 \mathrm{~km}$. Due to these similarities, simultaneous coverage of the facility is achieved at approximately $0^{\circ}$ to $60^{\circ}$ in elevation angle, as is apparent in Figure $13 \mathrm{c}$.

The 16-day $L_{1}$ and $L_{2}$ vertical orbit scenario also utilizes multiple libration point orbits (Figure 14). The periapsis altitudes are much larger than the near-rectilinear halo orbits at $48,600 \mathrm{~km}$ and $55,800 \mathrm{~km}$, respectively. The corresponding apoapsis altitudes are approximately $82,100 \mathrm{~km}$ for the $L_{1}$ vertical orbit and $69,600 \mathrm{~km}$ for the $L_{2}$ vertical orbit. Due to the nature of the "figure- 8 " shape, no two-spacecraft combination exhibits complete coverage of a south pole facility, as is apparent in Figure 14b. Neither spacecraft is within the line-of-sight of the facility when both spacecraft cross the fundamental plane at the same time. In fact, the STK results in Figure 14 a reveal an instant when no access beams (yellow) exist between the facility and either spacecraft. The STK elevation plot in Figure $14 \mathrm{c}$ also confirms that there are, in fact, small intervals of time when the facility does not possess line-of-sight to either spacecraft. An alternative scenario that employs the use of a vertical orbit for complete coverage is to place just one spacecraft in a 14-day $L_{1}$ vertical orbit and the other in a 14-day $L_{2}$ halo orbit.

The orbits for two spacecraft in a single 14-day $L_{2}$ butterfly orbit appear in Figure 15 . As with the near-rectilinear halo orbit scenarios, motion along a butterfly orbit remains primarily within direct line-ofsight of the facility throughout the orbit with a low periapsis altitude of approximately $8,800 \mathrm{~km}$. The corresponding apoapsis altitude for this orbit is approximately $67,900 \mathrm{~km}$. However, these orbits possess a much greater stability index than that of the near-rectilinear halo orbits. As seen in Figure $15 \mathrm{~b}$, the GENERATOR minimum $z$-displacement crossing occurs $36,000 \mathrm{~km}$ below the fundamental plane. Figure $15 \mathrm{c}$ demonstrates that at least one spacecraft is always $45^{\circ}$ above the horizon.

Finally, Figure 16 a depicts a unique 14-day $L_{2}$ butterfly orbit and 7-day $L_{2}$ halo orbit scenario utilizing two different orbits from two different families. The scenario also exploits a 2:1 ratio between periods. For this specific scenario, the minimum $z$-displacement crossover occurs $45,000 \mathrm{~km}$ below the fundamental plane (Figure 16b). Thus, simultaneous coverage occurs over elevation angles $0^{\circ}$ to $50^{\circ}$ (Figure 16c).

\section{Station-Keeping Results for Orbits Investigated}

Another important factor in determining architectures for lunar south pole coverage is the stationkeeping cost associated with each spacecraft. Station-keeping results are obtained in Astrogator Connect using the algorithm represented in Figure 7. Results for each orbit in Table 1 are specified in Table 2 for one complete year. In general, station-keeping cost increases with stability index. The lowest costs correspond to the 7-and 8-day $L_{2}$ near-rectilinear halo orbits while the highest costs are associated with the 14-day $L_{1}$ vertical orbit. Recall that these costs have not been optimized.

Table 2

STATION-KEEPING RESULTS FOR ONE YEAR ( 24 REVS)

\begin{tabular}{|c|c|c|c|c|c|c|c|}
\hline Orbit Type & $\begin{array}{l}\text { Libration } \\
\text { Point }\end{array}$ & $\begin{array}{l}\text { Period } \\
\text { (days) }\end{array}$ & $\begin{array}{c}\text { Avg. } 3 \sigma \\
\Delta v(\mathrm{~cm} / \mathrm{s})\end{array}$ & $\begin{array}{c}\text { No. of } \\
\text { Maneuvers }\end{array}$ & $\begin{array}{l}\text { Avg. Time Between } \\
\text { Maneuvers (days) }\end{array}$ & $\begin{array}{c}\text { Avg. } \Delta v \\
(\mathrm{~m} / \mathrm{s})\end{array}$ & $\begin{array}{l}\text { Total } \Delta y \\
(\mathrm{~m} / \mathrm{s})\end{array}$ \\
\hline Near-Rectilinear Halo & $L_{2}$ & 7.0 & 2.06 & 86 & 4.20 & 0.057 & 4.82 \\
\hline Near-Rectilinear Halo & $L_{1}$ & 8.0 & 1.52 & 55 & 6.40 & 0.101 & 5.54 \\
\hline Near-Rectilinear Halo & $L_{2}$ & 8.0 & 2.18 & 55 & 6.40 & 0.086 & 4.69 \\
\hline Halo & $L_{1}$ & 12.0 & 3.82 & 60 & 5.00 & 1.106 & 66.33 \\
\hline Halo & $L_{2}$ & 14.0 & 2.77 & 156 & 2.33 & 0.183 & 28.47 \\
\hline Vertical & $L_{\mathrm{t}}$ & 14.0 & 3.13 & 68 & 5.25 & 2.527 & 171.82 \\
\hline Butterfly & $L_{2}$ & 14.0 & 9.78 & 78 & 4.67 & 0.409 & 31.86 \\
\hline Vertical & $L_{1}$ & 16.0 & 2.81 & 91 & 4.00 & 0.347 & 31.55 \\
\hline Vertical & $L_{3}$ & 16.0 & 2.75 & 60 & 6.00 & 1.472 & 88.32 \\
\hline
\end{tabular}



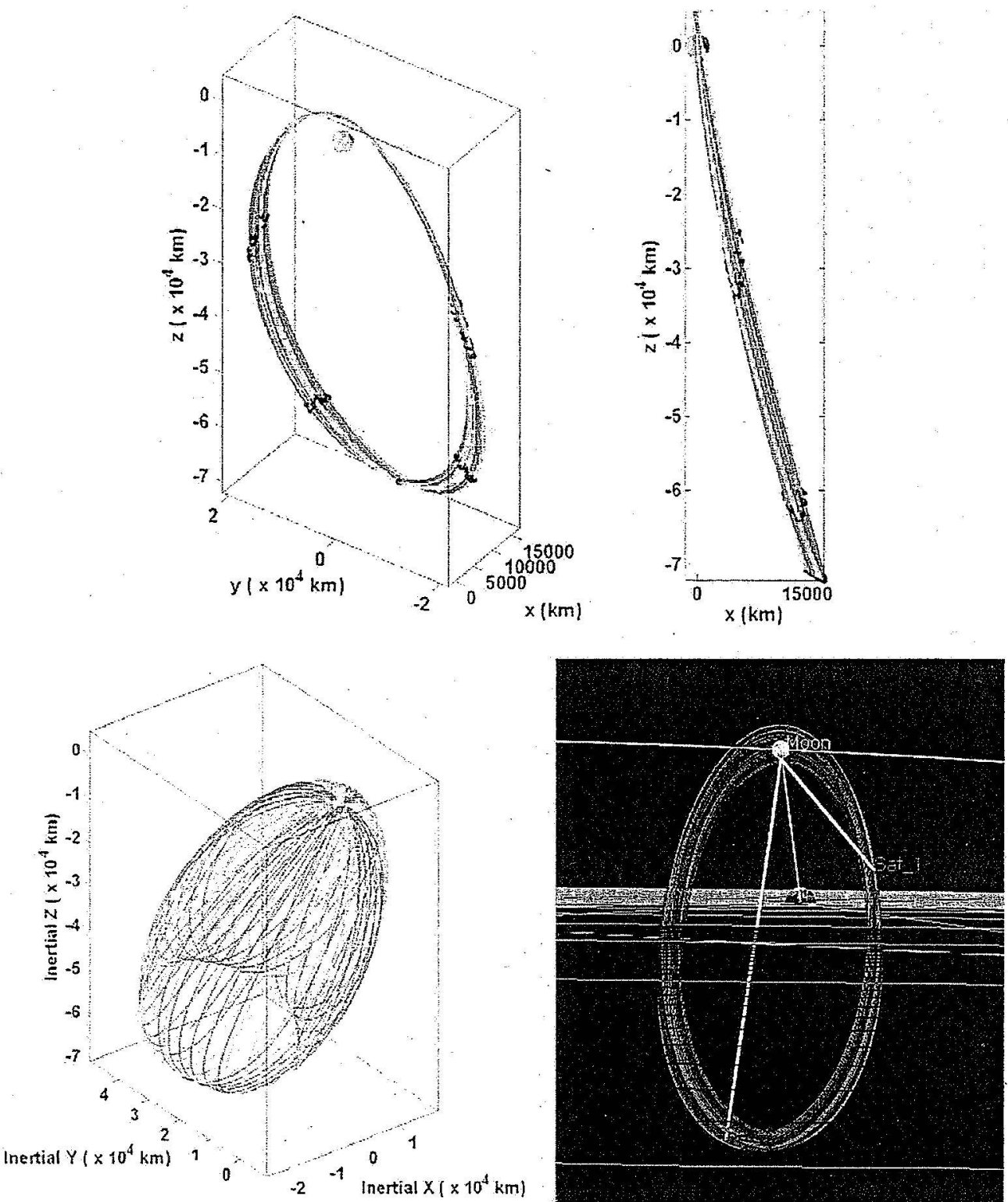

Figure 12a Two Phased Spacecraft in 7-Day $L_{2}$ Halo Orbits:

Moon Centered, Rotating Reference Frame (Top Left), $X-Z$ Projection (Top Right);

Inertial Mean J2000 Reference Frame (Bottom Left) in GENERATOR; Access Beams in STK Earth Centered, Rotating Reference Frame (Bottom Right) 


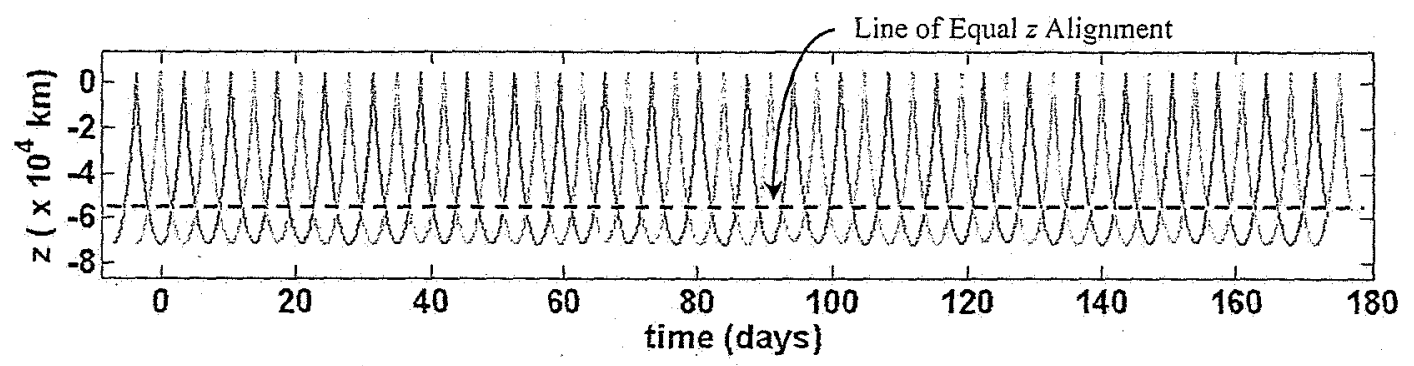

Figure $12 b \quad$-Displacement via GENERATOR for 7-Day $L_{2}$ Halo Orbit Scenario

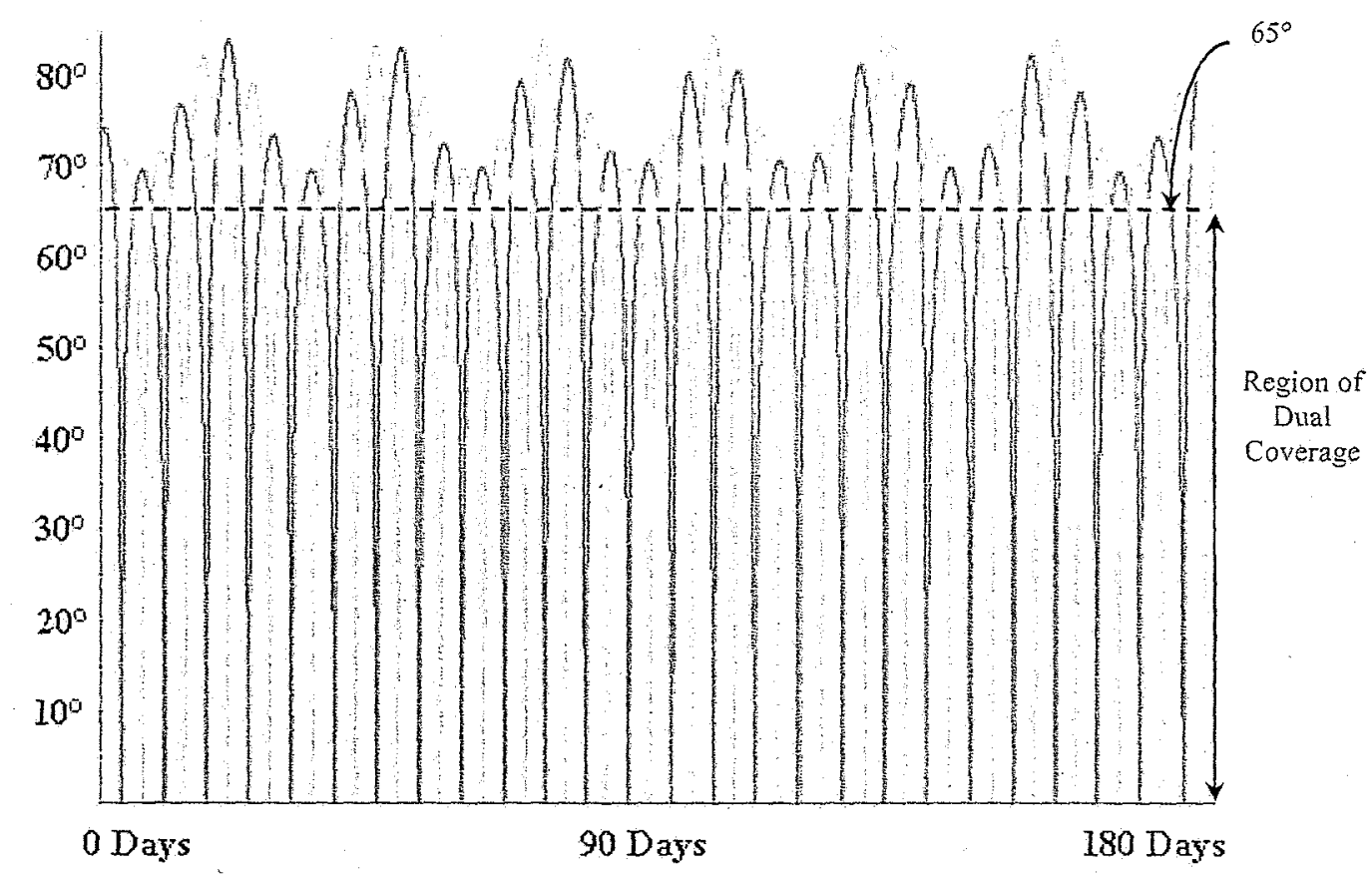

Figure 12c Elevation of Spacecraft via STK from Lunar South Pole Facility via STK for 7-Day $L_{2}$ Halo Orbit Scenario 

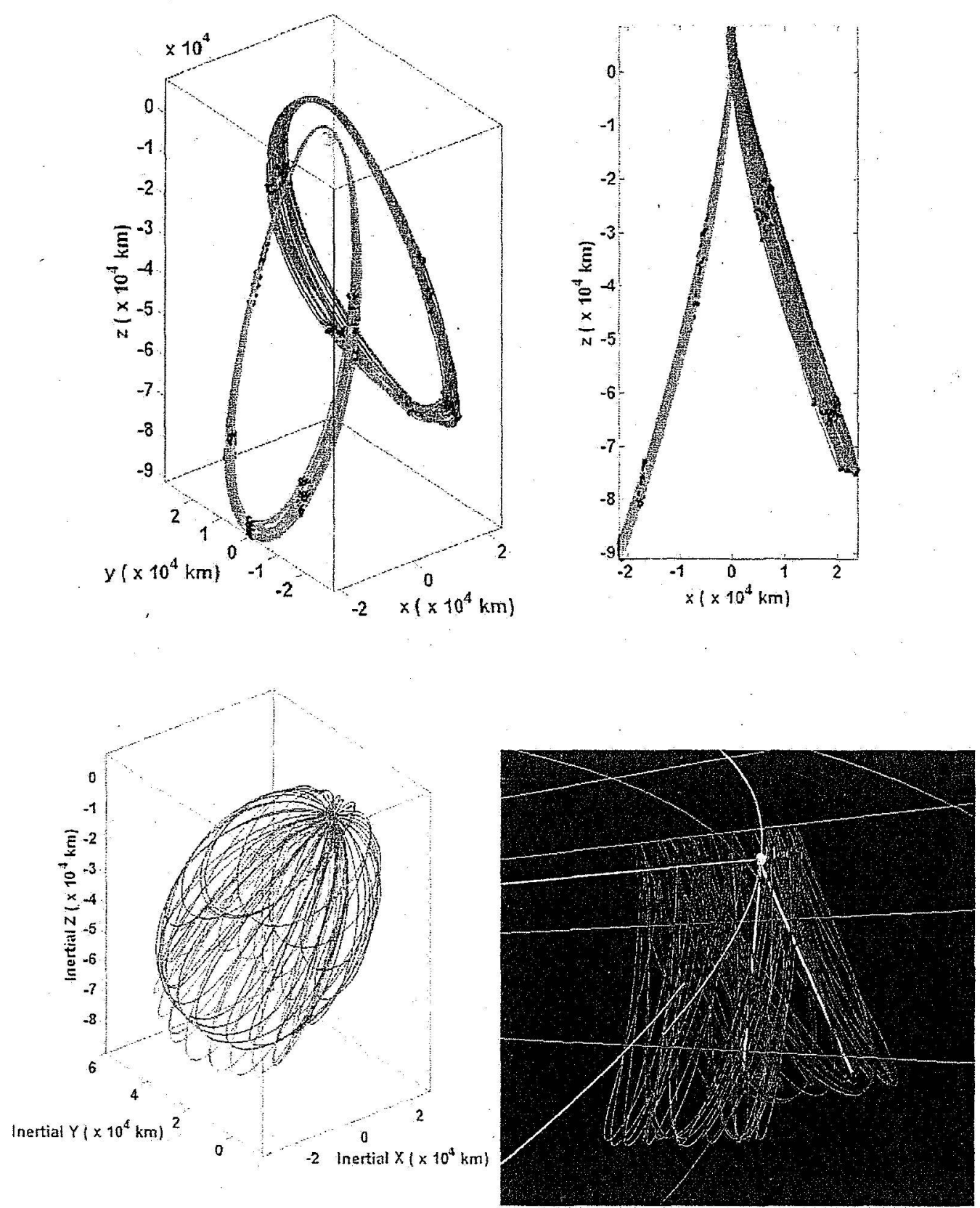

Figure 13a Two Phased Spacecraft in 8-Day $L_{1}$ (red) and $L_{2}$ (blue) Halo Orbits: Moon Centered, Rotating Reference Frame (Top Left), $X-Z$ Projection (Top Right); Inertial Mean J2000 Reference Frame (Bottom Left) in GENERATOR; Access Beams in STK Earth Centered, Rotating Reference Frame (Bottom Right) 


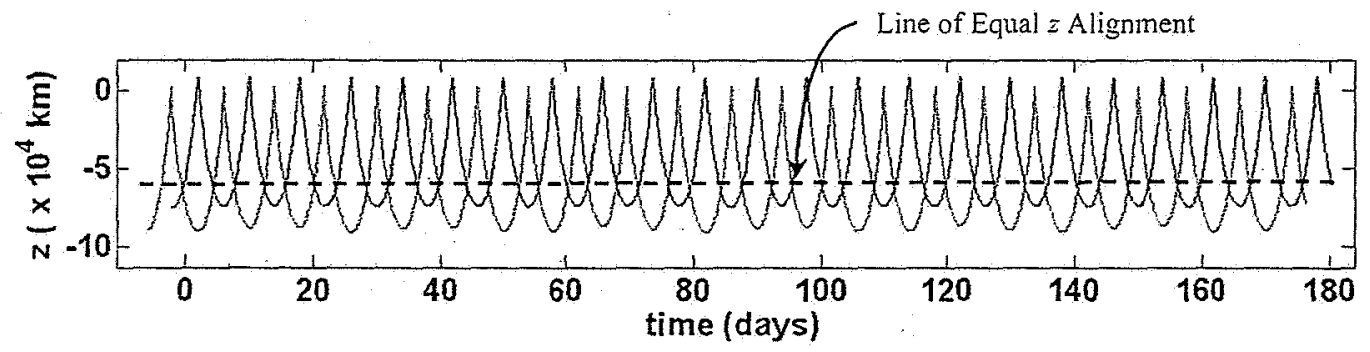

Figure 13b z-Displacement via GENERATOR for 8-Day $L_{1}$ (red) and $L_{2}$ (blue) Halo Orbit Scenario

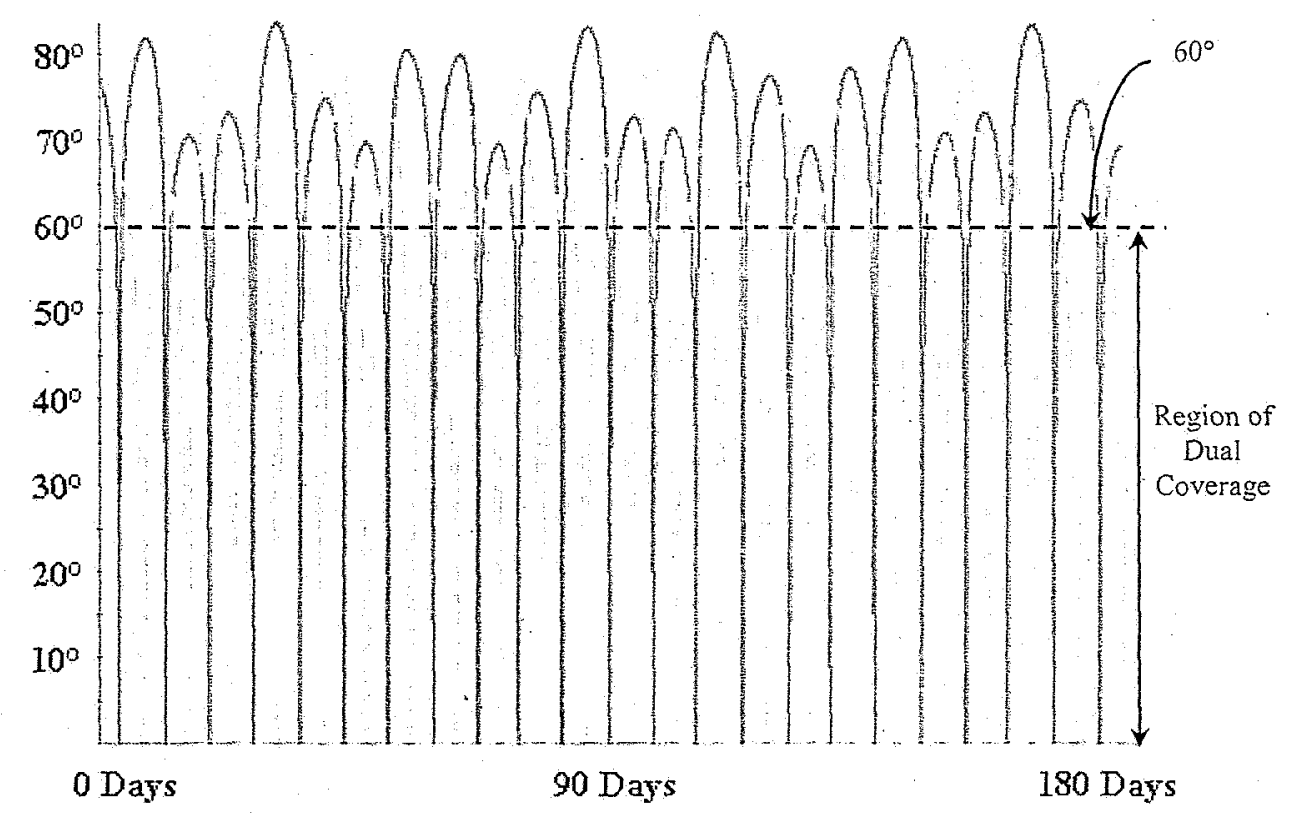

Figure 13c Elevation of Spacecraft via STK from Lunar South Pole Facility for 8-Day $L_{I}$ and $L_{2}$ Halo Orbit Scenario 

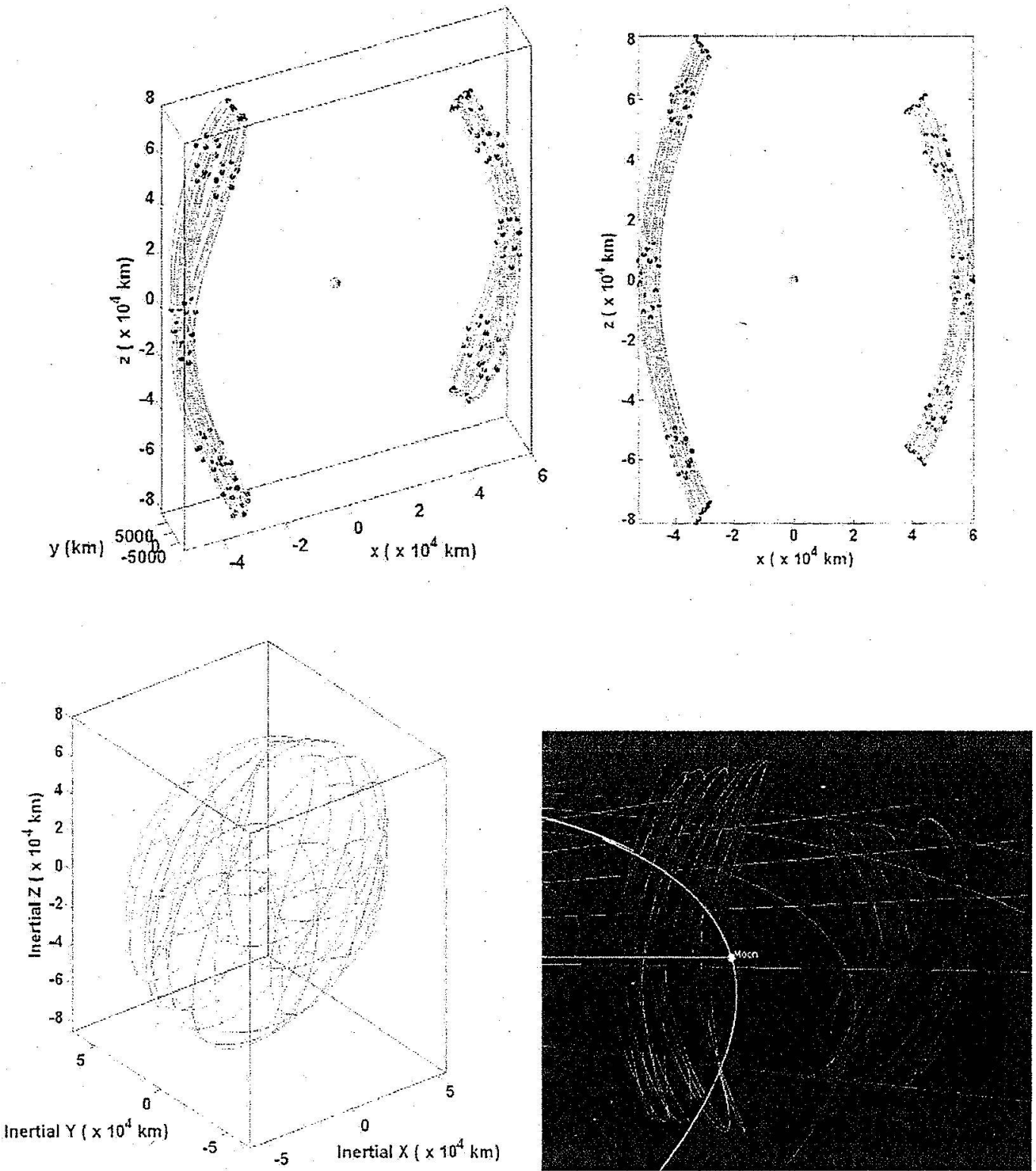

Figure 14a Two Phased Spacecraft in 16-Day $L_{1}$ (red) and $L_{2}$ (blue) Vertical Orbits: Moon Centered, Rotating Reference Frame (Top Left), $X-Z$ Projection (Top Right); Inertial Mean J2000 Reference Frame (Bottom Left) in GENERATOR; STK Earth Centered Rotating, Reference Frame (Bottom Right) 


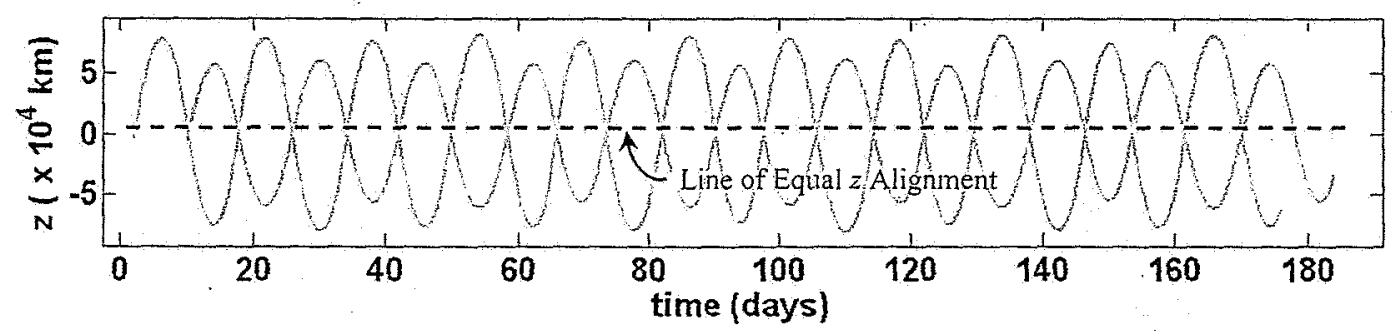

Figure 14b $\quad z$-Displacement via GENERATOR for 16-Day $L_{1}$ and $L_{2}$ Vertical Orbit Scenario

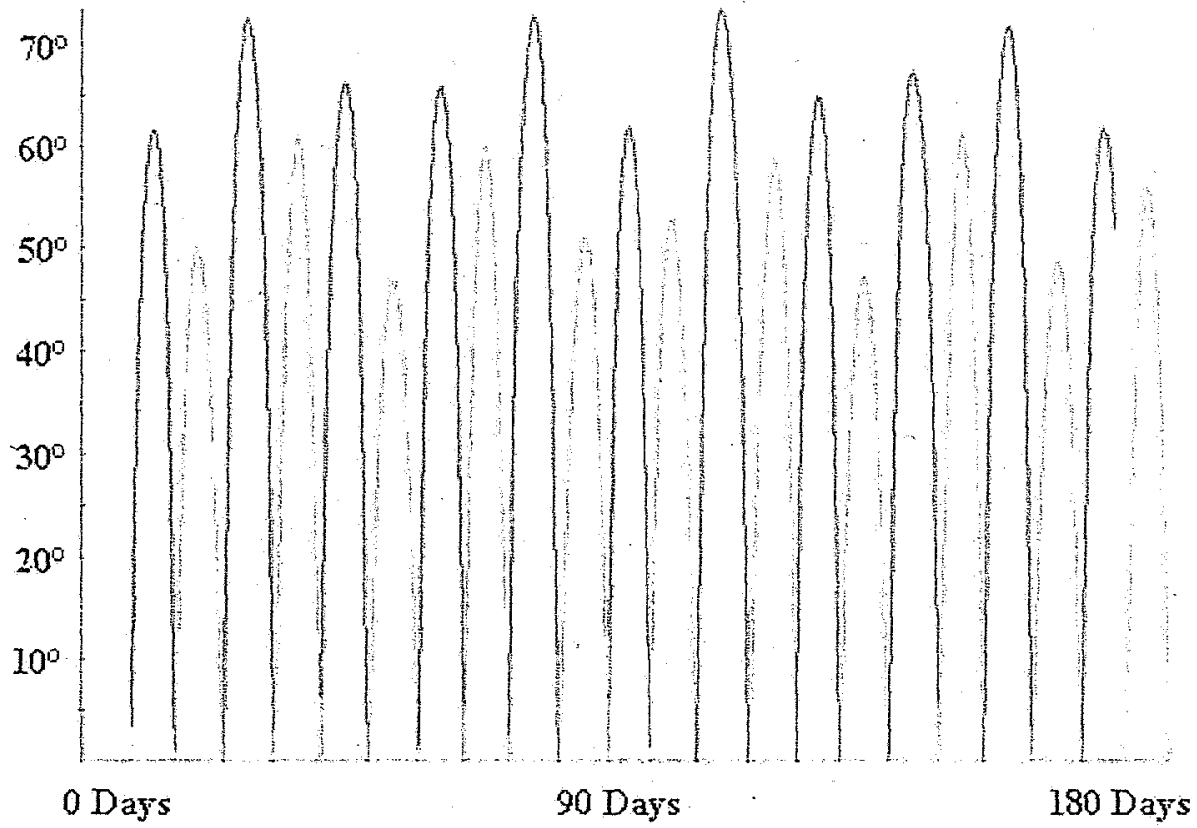

Figure 14c Elevation of Spacecraft via STK from Lunar South Pole Facility for 16-Day $L_{z}$ Halo Orbit Scenario 

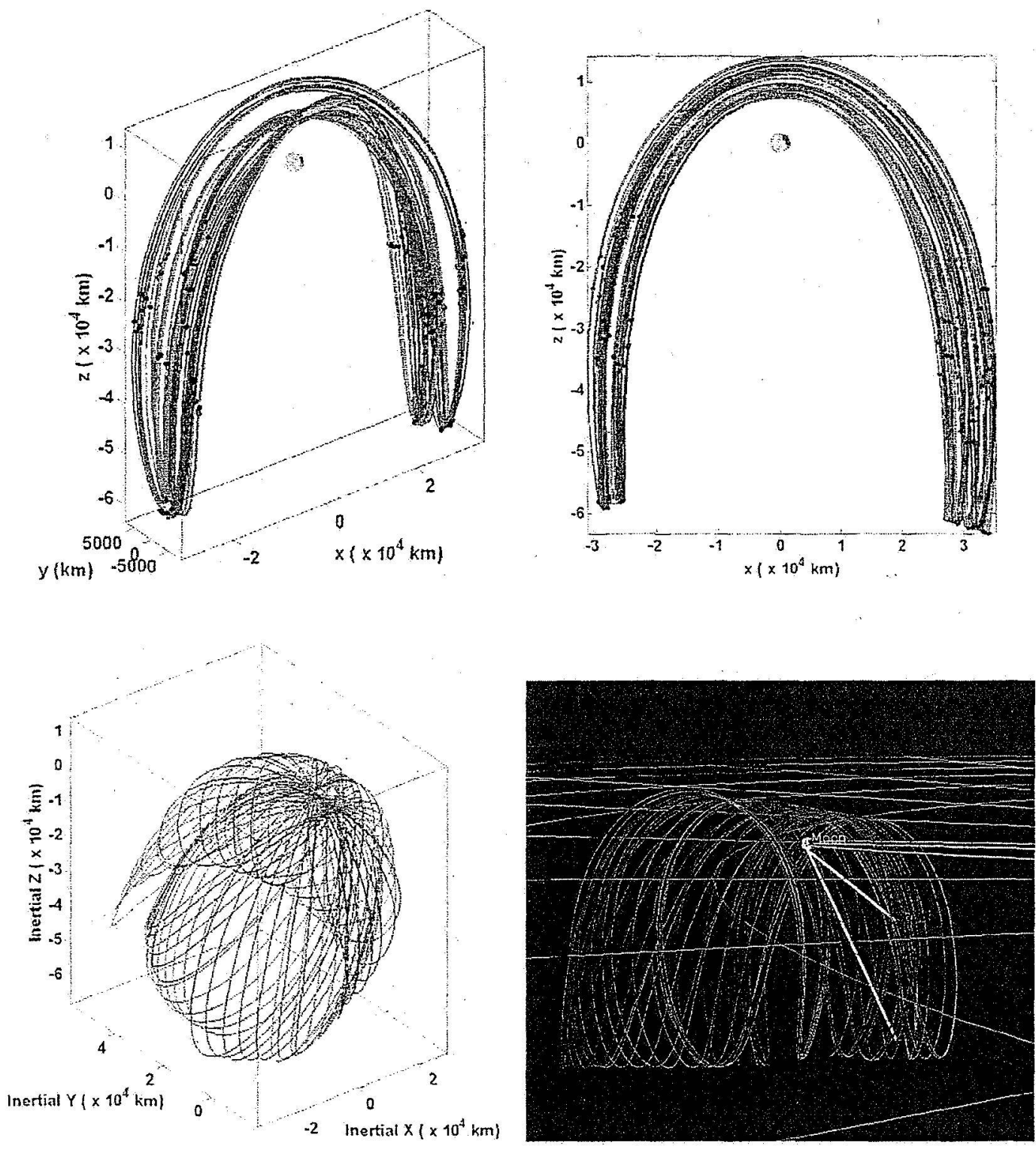

Figure 15a Two Phased Spacecraft in 14-Day $L_{2}$ Butterfly Orbits:

Moon Centered, Rotating Reference Frame (Top Left), $X-Z$ Projection (Top Right);

Inertial Mean J2000 Reference Frame (Bottom Left) in GENERATOR; Access Beams in STK Earth Centered, Rotating Reference Frame (Bottom Right) 


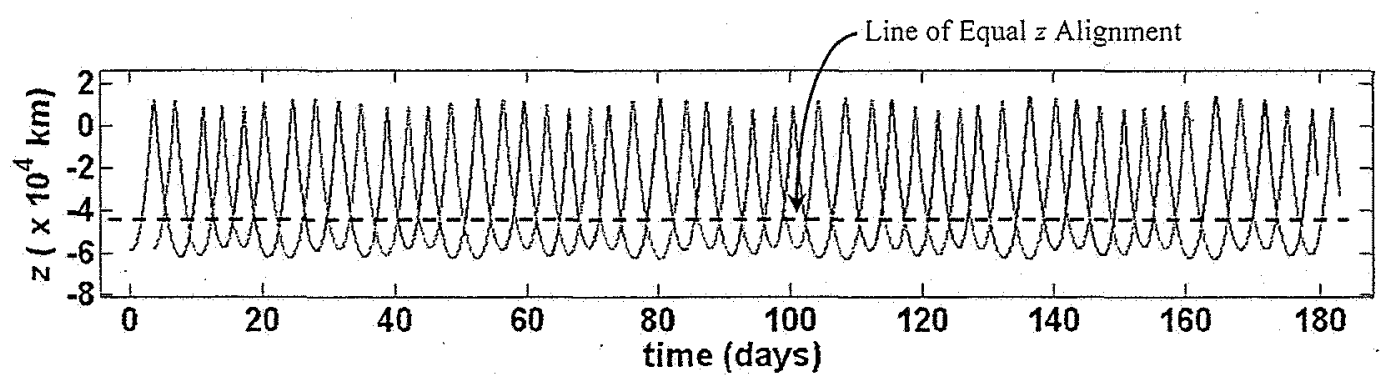

Figure 15b $z$-Displacement via GENERATOR for 14-Day $L_{2}$ Butterfly Orbit Scenario

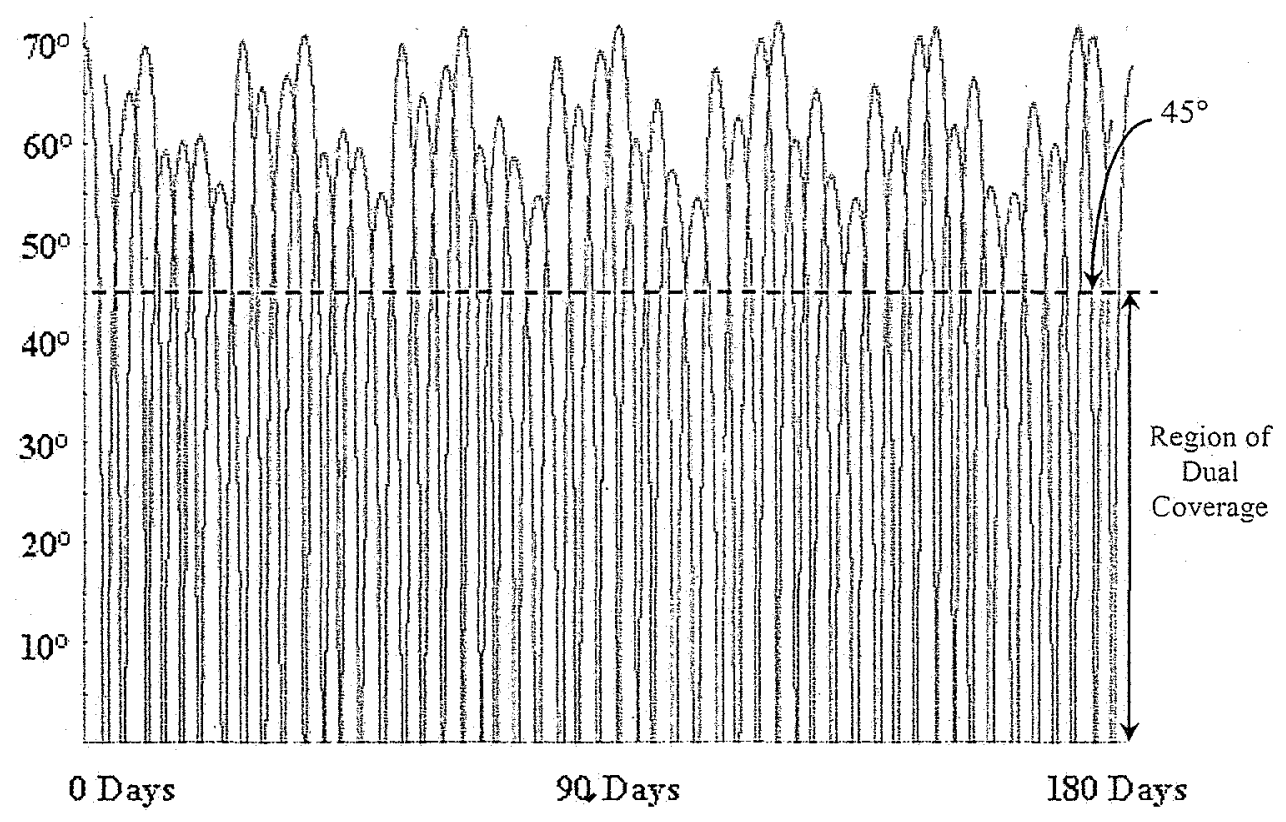

Figure 15c Elevation of Spacecraft via STK from Lunar South Pole Facility for 14-Day $L_{2}$ Butterfly Orbit Scenario 

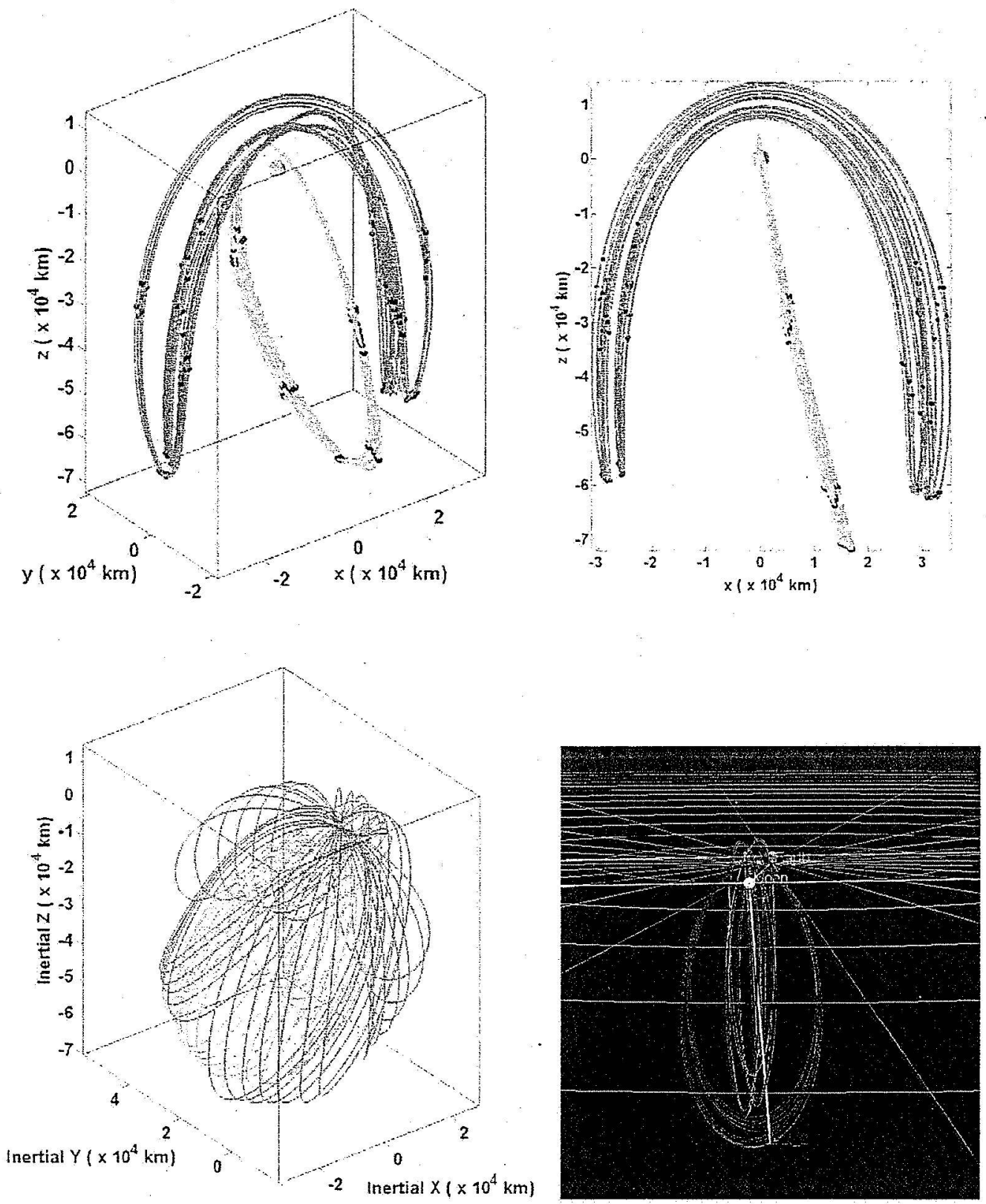

Figure 16a Two Spacecraft in 7-Day $L_{2}$ Halo Orbit (red) and a 14-day $L_{2}$ (blue) Butterfly Orbit: Moon Centered, Rotating Reference Frame (Top Left), $X-Z$ Projection (Top Right);

Inertial Mean J2000 Reference Frame (Bottom Left) in GENERATOR; Access Beams in STK Earth Centered, Rotating Reference Frame (Bottom Right) 


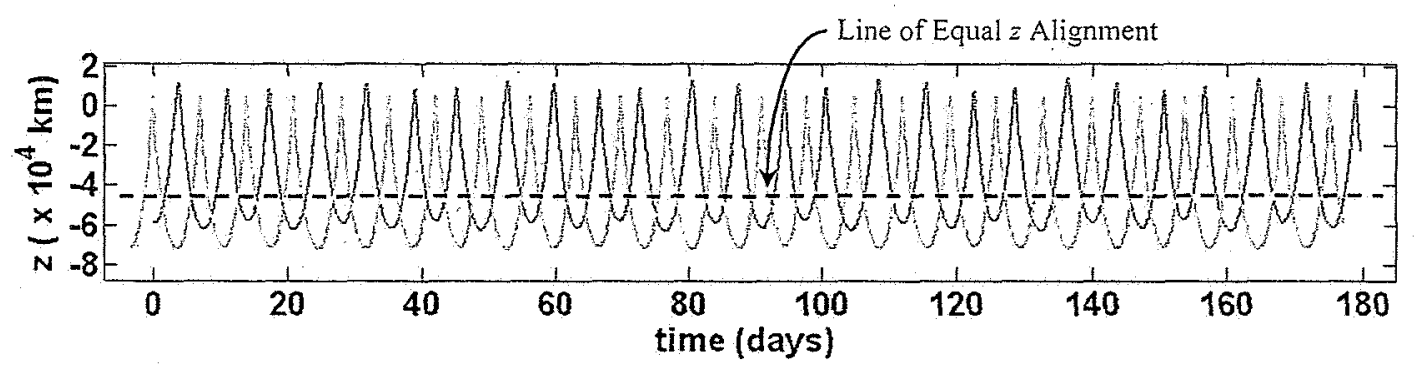

Figure $16 \mathrm{~b} \quad$-Displacement via GENERATOR for 7-Day $L_{2}$ Halo Orbit and a 14-day $L_{2}$ Butterfly Orbit Scenario

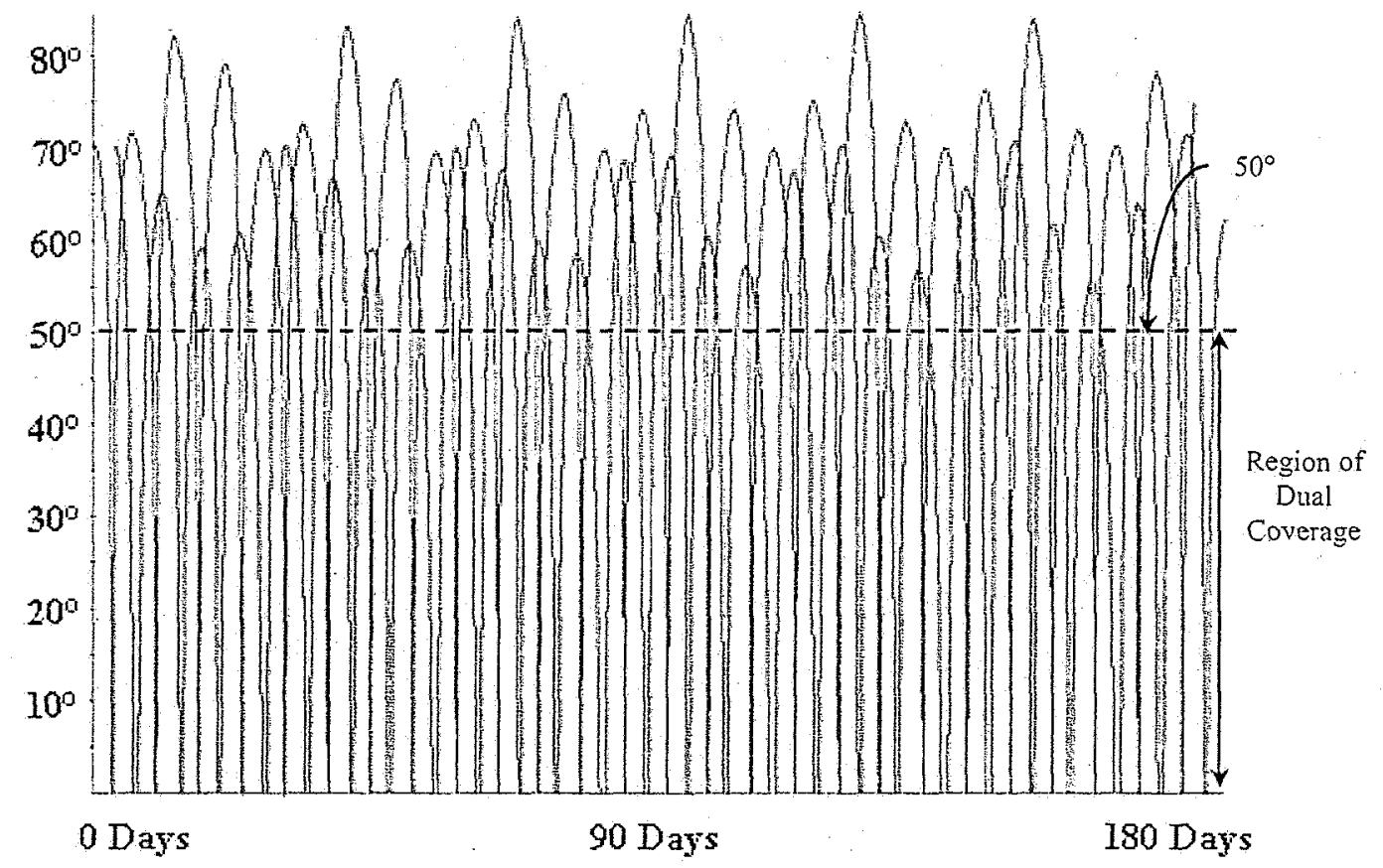

Figure 16c Elevation of Spacecraft via STK from Lunar Sonth Pole Facility 7-Day $L_{2}$ Halo Orbit and a 14-day $L_{2}$ Butterfly Orbit Scenario 


\section{CONCLUSION}

Orbits from the halo, vertical, and butterfly families in the vicinity of the Earth-Moon $L_{1}$ and $L_{2}$ libration points are selected in the CR3BP for potential lunar south pole coverage options. Orbits with altitudes between 50 and $100,000 \mathrm{~km}$ are identified as feasible. Nine different orbits with periods ranging from 7 to 16 days are studied in detail. Two phased spacecraft in a single orbit or in a combination of two different orbits, with periods that are commensurate, ensure adequate coverage of the lunar south pole. The orbit with the desired period is determined in the CR3BP from a corrections process with a periodicity constraint. Multiple revolutions are added and the orbit from the CR3BP is translated to a full ephemeris model, including solar perturbations, using the Purdue software GENERATOR. Quasi-periodic orbits from GENERATOR are attainable in STK with only minimal corrections for a complete coverage analysis of the lunar south pole. A preliminary station-keeping analysis is available using Astrogator Connect by targeting points along the baseline orbit and perturbing the spacecraft in a direction consistent with the unstable subspace.

Analysis continues to obtain transfers from the Earth to such orbits. Examples of the stable/unstable manifolds corresponding to these orbits appears in Figures 17 and 18. In general, the stability index offers information regarding the relative transfer costs of the orbits. In addition, libration point orbits from other families may also render coverage options for the lunar south pole. This procedure is also easily adaptable for designing feasible architectures for coverage of the north pole. Ultimately, each architecture possesses unique coverage characteristics, station-keeping cost, and transfer cost.

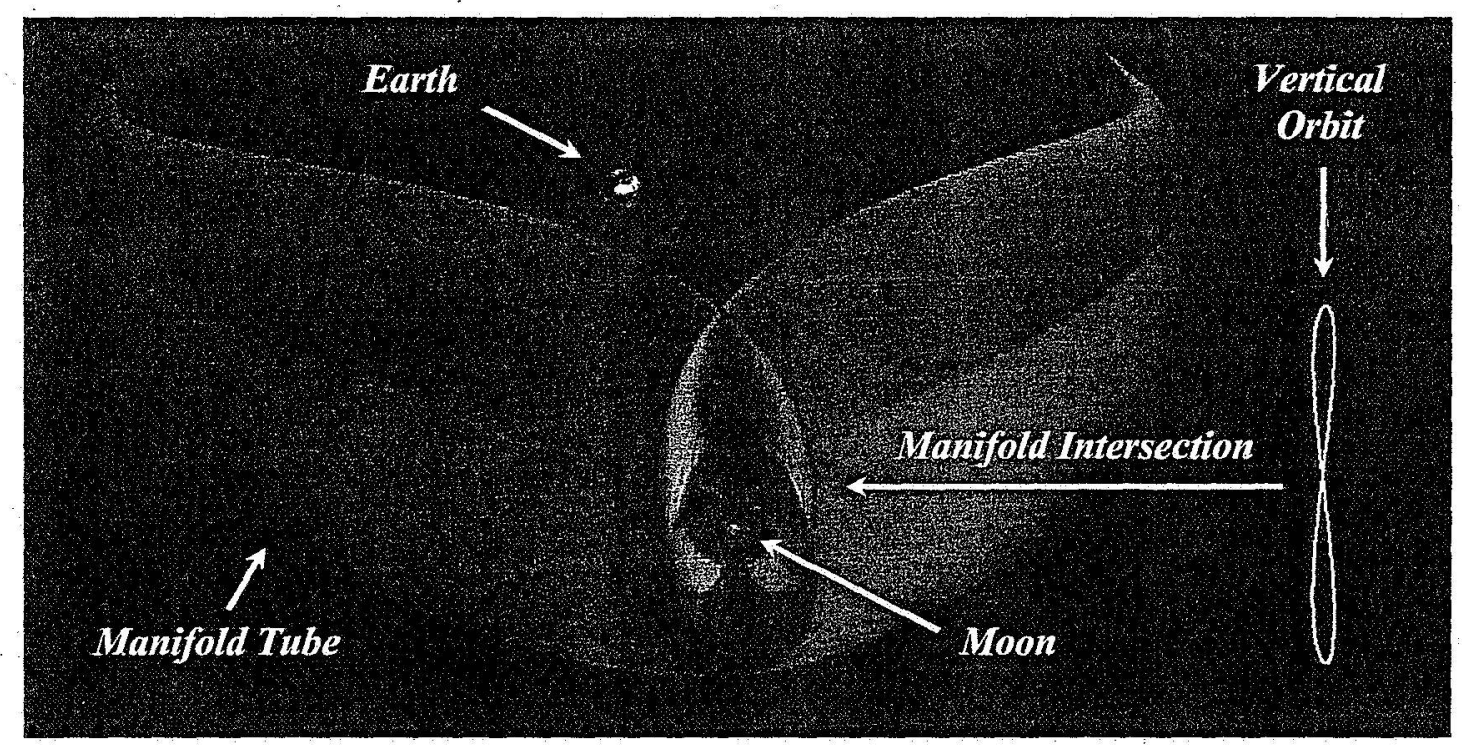

Figure $17 L_{1}$ Vertical Orbit with Intersecting Unstable (Red) and Stable (Blue) Manifolds from/to Earth and Moon Vicinities: CR3BP, Earth-Moon Rotating Frame. 


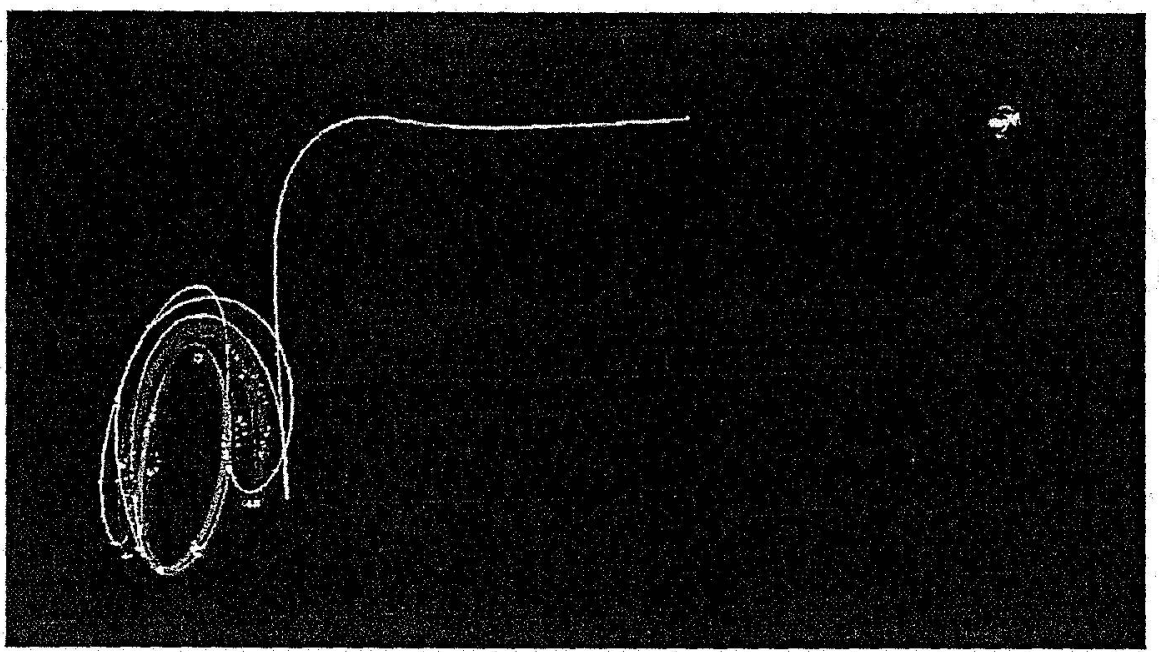

Figure 18 Two Spacecraft in 7-Day $L_{2}$ Halo Orbit (Magenta) and 14-Day $L_{2}$ Butterfly Orbit (Blue) with Stable Manifold (Green) from Earth Vicinity in Full Ephemeris Model Using GENERATOR.

\section{ACKNOWLEDGEMENT}

The authors would like to thank Frank Vaughn for the STK model adapted for this study. The majority of this work was carried out at the NASA Goddard Spaceflight Center with the Flight Dynamics and Analysis Branch. Portions of this were also supported by the GSFC NASA Academy and Purdue University.

\section{REFERENCES}

1. S. Nozette, C. Lichtenberg, P. Spudis, R. Bonner, W. Ort, E. Maleret, M. Robinson, and E. Shoemaker, "The Clementine Bistatic Radar Experiment." Science, Vol. 274, Issue 5292, 1996, pp. 1495-1498.

2. E. Kozlova, "The Presence of volatiles in the polar regions of the Moon." 35th COSPAR Scientific Assembly, Paris, France, 18-25 July 2004.

3. "The Vision for Space Exploration," National Aeronautics and Space Administration Publication, NP-2004-01334-HQ, February 2004.

4. NASA Space Science Enterprise, Strategic Plan. bitp//spacesciencenasa gov/admin/pubs/strategv/2000/, Sections 2-3, November 2000.

5. V. Szebehely, Theory of Orbits: The Restricted Problem of Three Bodies. New York: Academic Press, 1967.

6. C. Zagouras and P. Kazantzis, "Three-dimensional periodic oscillations generating from plane periodic ones around the collinear Lagrangian points." Astrophysics and Space Science, Vol. 61, No. 2, April 1979, pp. 389-409.

7. J. Breakwell and J. Brown, "The 'Halo' Family of 3-Dimensional Periodic orbits in the Earth-Moon Restricted 3Body Problem." Celestial Mechanics, Vol. 20, 1979, pp. 389-404.

8. I. Robin and V. Markellos, "Numerical Determinations of three-dimensional orbits generated from yertical selfresonant satellite orbits." Celestial Mechanics, Nov. 21, May 1980, pp. 395-434.

9. K. Howell and J. Breakwell, "Almost Rectilinear Halo Orbits." Celestial Mechanics, Vol. 32, No. 1, January 1984, p. 29-52. 
10. D. Dichman, E. Doedel, and R. Paffenroth, "The Computation of Periodic Solutions of the 3-Body Problem Using the Numerical Contimation Sofware AUTO." Libration Point Orbits and Applications, G. Gómez, M. Lo, J. Masdemont (Editors), Hong Kong, World Scientific, 2003.

11. F. Moulton (in collaboration with D. Buchanan, T. Buck, F. Griffin, W. Longley, and W. MacMillan), "Periodic Orbits." Camegie Institution of Washington, Washington, 1920.

12. R. Wilson and K. Howell, "Trajectory Design in the Sun-Earth-Moon System Using Multiple Lunar Gravity Assists." Joumal of Spacecraft and Rockets, Vol. 35, No. 2, March-April 1998, pp. 19i-198.

13. K. Howell and J. Anderson, User's Guide: Purdue Software GENERATOR, July 2001.

14. T. Ely, "Stable Constellations of Frozen Elliptical Inclined Orbits." Journal of the Astronautical Sciences, Vol. 53, No. 3, July-September 2005.

15. T. Ely and E. Lieb, "Constellations of Elliptical Inclined Lunar Orbits Providing Polar and Global Coverage." Paper No. AAS 05-158, AAS/AIAA Spaceflight Mechanics Meeting, Sonth Lake Tahoe, California, August 7-11, 2005.

16. R. Farquhar, "The Utilization of Flalo Orbits in Advanced Lunar Operations." NASA TND-365, GSFC, Greenbelt, Maryland, 1971.

17. C. Simó, G. Gómez, J. Libre, and R. Martinez, "Station Keeping of a Quasiperiodic Halo Orbit Using Invariant Manifolds." Proceedings of the Second International Symposium on Spaceflight Dynamics, Darmstadt; Germany, October 1986, pp. 65-70.

18. C. Simó, G. Gómez, J. Libre, R. Martínez, and J. Rodríguez, "On the Optimal Station Keeping Control of Halo Orbits." Acta Astonautica, Vol. 15, No. 677, 1987, pp.391-19.7.

19. K. Howell and H. Pernicka, "Stationkeeping Method for Libration Point Trajectories." Journal of Guidance and Control, Vol. 16, No. 1, 1993, pp. 151-159.

20. K. Howell and T. Keeter, "Station-Keeping Strategies for Libration Point Orbits: Target Point and Floquet Mode Approaches," AAS/AIAA Spaceflight Mechanics 1995. Advances in the Astronautical Sciences, Vol. 89, R. Proulx, J. Liu, P. Seidelmann, and S. Alfano (editors), 1995, pp. 1377.1396.

21. G. Gómez, K. Howell, J. Masdemont, and C. Simó, "Station-Keeping Strategies for Translunar Libration Point Orbits." Paper No. AAS 98-168, AAS/AIAA Spaceflight Mechanics Meeting, Monterey, California, Febriary 9$11,1998$.

22. D. Scheeres, D. Han, and Y. Hou, "The Influence of Unstable Manifolds on Orbit Uncertainty." Journal of Guidance, Control, and Dynamics, Vol. 24, No. 3. May-June 2001, pp. 573-585.

23. C. Renault and D. Scheeres, "Statistical Analysis of Control Maneuvers in Unstable Orbital Environments." Joumal of Guidance, Control, and Dynamics, Vol. 26, No. 5, September-October 2003, pp.758-769.

24. D. Folta and F. Vaughn, "A Survey of Earth-Moon Libration Orbits: Station-Keeping Strategies and Intra-Orbit Transfers." Proceedings of AAS/AIAA Astrodynamics Specialist Conference, Providence, Rhode Island, 2004.

25. G. Gómez, J. Libre, R. Martinez, and C. Simó, Dynamics and Mission Design Near Libration Points; Vol. I, Fundamentals: The Case of Collinear Libration Points. Singapore: World Scientific Publishing Co. Pte. Ltd., 2001.

26. K. Howell and H. Pemicka, "Numerical Determination of Lissajous Trajectories in the Restricted Three-Body Problem." Celestial Mechanics, Vol.41, 1988, pp. 107-124.

27. S. Hughes, D. Cooley, and J. Guzman, "A Direct Method for Fuel Optimal Maneuvers of Distributed Spacecraft in Multiple Flight Regimes." Paper No. AAS 05-158, Space Flight Mechanics Meeting, Copper Mountain, Colorado, January 23-27, 2005. 
28. B. Marchand, K. Howell, and R. Wilson, "A Trajectory Design Strategy in the Multi-Body Regime Including Constraints." (in prep)

29. B. Marchand and K. Howell, "Aspherical Formations Near the Libration Points in the Sun-Earth/Moon Ephemeris System." Paper No. AAS 04-157, 14th AAS/AIAA Space Flight Mechanics Conference, Maui, Hawaii, February $8-12,2004$. 